\title{
ON THE CLASSICAL STRING SOLUTIONS AND STRING/FIELD THEORY DUALITY II
}

\author{
D. Aleksandrova, P. Bozhilov \\ Department of Theoretical and Applied Physics, \\ Shoumen University, 9712 Shoumen, Bulgaria \\ E-mail: p.bozhilov@shu-bg.net
}

\begin{abstract}
Based on the recently considered classical string configurations, in the framework of the semi-classical limit of the string/gauge theory correspondence, we describe a procedure for obtaining exact classical string solutions in general string theory backgrounds, when the string embedding coordinates depend non-linearly on the worldsheet spatial parameter. The tensionless limit, corresponding to small t'Hooft coupling on the field theory side, is also considered. Applying the developed approach, we first reproduce some known results. Then, we find new string solutions - with two spins in $A d S_{5}$ black hole background and in $A d S_{5} \times S^{5}$ with two spins and up to nine independent conserved $R$-charges.
\end{abstract}

PACS codes: 11.25.-w, 11.27.+d, 11.30.-j

Keywords: Bosonic Strings, AdS-CFT and dS-CFT Correspondence, Space-Time Symmetries, Integrable Equations in Physics.

\section{Introduction}

The article [1] on the semi-classical limit of the string/gauge theory duality initiated an interest in the investigation of the connection between the classical string solutions, their semi-classical quantization and the string/field theory correspondence [2] - [27 ${ }^{1}$. Most of the papers consider different string configurations in type IIB $A d S_{5} \times S^{5}$ background. However, the string dynamics has been investigated in other string theory backgrounds, known to have field theory dual descriptions in different dimensions, with different number of (or without) supersymmetries, conformal or non-conformal.

For establishing the correspondence between the semi-classically quantized string solutions and the appropriate objects in the dual field theory, it is essential for one to know the explicit expressions for the conserved quantities like energy, angular momentum, etc., on the string theory side. Their existence is connected with the symmetries of the corresponding supergravity backgrounds. The analysis of the connection between the ansatzes, used to obtain exact string solutions, and the background symmetries shows

\footnotetext{
${ }^{1}$ For earlier studies on the subject, see [28, 29] and the references in [29] and [30].
} 
that the string embeddings can be divided into the following four types [31]:

$$
\begin{array}{r}
X^{\mu}(\tau, \sigma)=\Lambda_{0}^{\mu} \tau+\Lambda_{1}^{\mu} \sigma, \quad X^{a}(\tau, \sigma)=Y^{a}(\tau) \\
X^{\mu}(\tau, \sigma)=\Lambda_{0}^{\mu} \tau+\Lambda_{1}^{\mu} \sigma+Y^{\mu}(\tau), \quad X^{a}(\tau, \sigma)=Y^{a}(\tau) \\
X^{\mu}(\tau, \sigma)=\Lambda_{0}^{\mu} \tau+\Lambda_{1}^{\mu} \sigma, \quad X^{a}(\tau, \sigma)=Z^{a}(\sigma) \\
X^{\mu}(\tau, \sigma)=\Lambda_{0}^{\mu} \tau+\Lambda_{1}^{\mu} \sigma+Z^{\mu}(\sigma), \quad X^{a}(\tau, \sigma)=Z^{a}(\sigma) \\
\Lambda_{m}^{\mu}=\text { const }, \quad(m=0,1) .
\end{array}
$$

Here, the string embedding coordinates $X^{M}(\tau, \sigma),(M=0,1, \ldots, D-1)$, are represented as $X^{M}=\left(X^{\mu}, X^{a}\right)$. $X^{\mu}(\tau, \sigma)$ correspond to the space-time coordinates $x^{\mu}$, on which the background fields do not depend. This means that there exist $n_{\mu}$ commuting Killing vectors $\partial / \partial x^{\mu}$, where $n_{\mu}$ is the number of the coordinates $x^{\mu}$.

All the ansatzes used in [1 - 27] for the string embedding, are particular cases of (1.1)

- (1.4), except those in [16, 27] ${ }^{2}$. In [6, 9, 14, 22] there are of type (1.1), in [1] and [7] - of type (1.2). In [1] - [5], 7], [8], [10, [1], 14] - [20, [23] - [26], ansatzes of type (1.3) are considered. Solutions, based on the ansatzes of type (1.4), are obtained in [12 - 14, [21.

The aim of this article is to describe a procedure for obtaining the exact classical string solutions in general string theory backgrounds, based on the ansatzes (1.3) and (1.4) ${ }^{3}$. We will use more general worldsheet gauge than the conformal one, in order to be able to discuss the tensionless limit $T \rightarrow 0$, corresponding to small t'Hooft coupling $\lambda \rightarrow 0$ on the field theory side.

Let us also note that in (1.1)-(1.4), we have separated the cases $Y^{\mu}=0$ and $Y^{\mu} \neq 0$, $Z^{\mu}=0$ and $Z^{\mu} \neq 0$, because the types of the string solutions in these cases are essentially different, as we will see later on.

The paper is organized as follows. In Sec.2 and Sec.3, we describe the string dynamics and give the corresponding exact solutions of the equations of motion and constraints, based on the ansatzes (1.3) and (1.4) respectively. Sec.4 is devoted to some applications of the derived general results. In Sec. 5, we conclude with several remarks.

\section{$2 \quad$ Exact string solutions}

In our further considerations, we will explore the Polyakov type action for the bosonic string in a $D$-dimensional curved space-time with metric tensor $g_{M N}(x)$, interacting with a background 2-form gauge field $b_{M N}(x)$ via Wess-Zumino term

$$
\begin{aligned}
& S^{P}=\int d^{2} \xi \mathcal{L}^{P}, \quad \mathcal{L}^{P}=-\frac{1}{2}\left(T \sqrt{-\gamma} \gamma^{m n} G_{m n}-Q \varepsilon^{m n} B_{m n}\right), \\
& \xi^{m}=\left(\xi^{0}, \xi^{1}\right)=(\tau, \sigma), \quad m, n=(0,1),
\end{aligned}
$$

where

$$
\begin{aligned}
& G_{m n}=\partial_{m} X^{M} \partial_{n} X^{N} g_{M N}, \quad B_{m n}=\partial_{m} X^{M} \partial_{n} X^{N} b_{M N} \\
& \left(\partial_{m}=\partial / \partial \xi^{m}, \quad M, N=0,1, \ldots, D-1\right)
\end{aligned}
$$

\footnotetext{
${ }^{2}$ See section 5 .

${ }^{3}$ The corresponding results, based on the ansatzes (1.1) and (1.2), can be found in [31.
} 
are the fields induced on the string worldsheet, $\gamma$ is the determinant of the auxiliary worldsheet metric $\gamma_{m n}$, and $\gamma^{m n}$ is its inverse. The position of the string in the background space-time is given by $x^{M}=X^{M}\left(\xi^{m}\right)$, and $T=1 / 2 \pi \alpha^{\prime}, Q$ are the string tension and charge, respectively. If we consider the action (2.1) as a bosonic part of a supersymmetric one, we have to put $Q= \pm T$. In what follows, $Q=T$.

The equations of motion for $X^{M}$ following from (2.1) are:

$$
\begin{aligned}
& -g_{L K}\left[\partial_{m}\left(\sqrt{-\gamma} \gamma^{m n} \partial_{n} X^{K}\right)+\sqrt{-\gamma} \gamma^{m n} \Gamma_{M N}^{K} \partial_{m} X^{M} \partial_{n} X^{N}\right] \\
& =\frac{1}{2} H_{L M N} \epsilon^{m n} \partial_{m} X^{M} \partial_{n} X^{N},
\end{aligned}
$$

where

$$
\begin{aligned}
& \Gamma_{L, M N}=g_{L K} \Gamma_{M N}^{K}=\frac{1}{2}\left(\partial_{M} g_{N L}+\partial_{N} g_{M L}-\partial_{L} g_{M N}\right), \\
& H_{L M N}=\partial_{L} b_{M N}+\partial_{M} b_{N L}+\partial_{N} b_{L M},
\end{aligned}
$$

are the components of the symmetric connection corresponding to the metric $g_{M N}$, and the field strength of the gauge field $b_{M N}$ respectively. The constraints are obtained by varying the action (2.1) with respect to $\gamma_{m n}$ :

$$
\delta_{\gamma_{m n}} S^{P}=0 \Rightarrow\left(\gamma^{k l} \gamma^{m n}-2 \gamma^{k m} \gamma^{l n}\right) G_{m n}=0 .
$$

Now, our task is to find exact solutions of the nonlinear differential equations (2.2) and (2.3). Let us first consider the constraints (2.3). We have three constraints in (2.3), but only two of them are independent. To extract the independent ones, we rewrite the three constraints as follows:

$$
\begin{aligned}
& \left(\gamma^{00} \gamma^{m n}-2 \gamma^{0 m} \gamma^{0 n}\right) G_{m n}=0, \\
& \left(\gamma^{01} \gamma^{m n}-2 \gamma^{0 m} \gamma^{1 n}\right) G_{m n}=0, \\
& \left(\gamma^{11} \gamma^{m n}-2 \gamma^{1 m} \gamma^{1 n}\right) G_{m n}=0 .
\end{aligned}
$$

Inserting $G_{11}$ from (2.6) into (2.4) and (2.5), one obtains that both of them are satisfied, when the equality

$$
\gamma^{01} G_{00}+\gamma^{11} G_{01}=0
$$

holds. To simplify the constraint (2.6), we put (2.7) in it, which results in

$$
\gamma^{00} G_{00}-\gamma^{11} G_{11}=0 \text {. }
$$

Thus, our independent constraints, with which we will work from now on, are given by (2.7) and (2.8).

Now let us turn to the equations of motion (2.2). We will use the gauge $\gamma^{m n}=$ constants, in which they simplify to

$$
g_{L K} \gamma^{m n}\left(\partial_{m} \partial_{n} X^{K}+\Gamma_{M N}^{K} \partial_{m} X^{M} \partial_{n} X^{N}\right)=-\frac{1}{2 \sqrt{-\gamma}} H_{L M N} \epsilon^{m n} \partial_{m} X^{M} \partial_{n} X^{N} .
$$

In particular, $\gamma^{m n}=\eta^{m n}=\operatorname{diag}(-1,1)$ correspond to the commonly used conformal gauge. 


\subsection{Solving the equations of motion and constraints}

In solving the equations of motion and constraints, we will use string embedding that exploits the symmetries of the background. Namely, our ansatz for the string coordinates $X^{M}(\tau, \sigma)=\left(X^{\mu}, X^{a}\right)$ in this section is given by (1.3) , and $x^{\mu}$ are the target space-time coordinates, on which the background fields do not depend:

$$
\partial_{\mu} g_{M N}=0, \quad \partial_{\mu} b_{M N}=0
$$

Taking into account the ansatz (1.3) and under the conditions (2.10), one obtains the following reduced Lagrangian density, arising from the action (2.1) (the prime is used for $d / d \sigma)$

$$
\begin{array}{r}
\mathcal{L}^{A}(\sigma)=-\frac{T}{2} \sqrt{-\gamma}\left[\gamma^{11} g_{a b} Z^{\prime a} Z^{\prime b}+2\right. \\
\left(\gamma^{1 m} \Lambda_{m}^{\mu} g_{\mu a}-\frac{1}{\sqrt{-\gamma}} \Lambda_{0}^{\mu} b_{\mu a}\right) Z^{\prime a}+ \\
\left.+\gamma^{m n} \Lambda_{m}^{\mu} \Lambda_{n}^{\nu} g_{\mu \nu}-\frac{2}{\sqrt{-\gamma}} \Lambda_{0}^{\mu} \Lambda_{1}^{\nu} b_{\mu \nu}\right]
\end{array}
$$

where the fields induced on the string worldsheet are given by

$$
\begin{gathered}
G_{00}=\Lambda_{0}^{\mu} \Lambda_{0}^{\nu} g_{\mu \nu}, \quad G_{01}=\Lambda_{0}^{\mu}\left(g_{\mu a} Z^{\prime a}+\Lambda_{1}^{\nu} g_{\mu \nu}\right) \\
G_{11}=g_{a b} Z^{\prime a} Z^{\prime b}+2 \Lambda_{1}^{\mu} g_{\mu a} Z^{\prime a}+\Lambda_{1}^{\mu} \Lambda_{1}^{\nu} g_{\mu \nu} \\
B_{01}=\Lambda_{0}^{\mu}\left(b_{\mu a} Z^{\prime a}+\Lambda_{1}^{\nu} b_{\mu \nu}\right)
\end{gathered}
$$

The constraints (2.8) and (2.7) respectively, and the equations of motion for $X^{M}$ (2.9), can be written as

$$
\begin{aligned}
& \gamma^{11}\left(g_{a b} Z^{\prime a} Z^{\prime b}+2 \Lambda_{1}^{\mu} g_{\mu b} Z^{\prime b}\right)-\left(\gamma^{00} \Lambda_{0}^{\mu} \Lambda_{0}^{\nu}-\gamma^{11} \Lambda_{1}^{\mu} \Lambda_{1}^{\nu}\right) g_{\mu \nu}=0 \\
& \Lambda_{0}^{\mu}\left(\gamma^{11} g_{\mu a} Z^{\prime a}+\gamma^{1 n} \Lambda_{n}^{\nu} g_{\mu \nu}\right)=0 \\
& \gamma^{11}\left(g_{L b} Z^{\prime \prime b}+\Gamma_{L, b c} Z^{\prime b} Z^{\prime c}\right)+2 \gamma^{1 m} \Lambda_{m}^{\mu} \Gamma_{L, \mu b} Z^{\prime b}+\gamma^{m n} \Lambda_{m}^{\mu} \Lambda_{n}^{\nu} \Gamma_{L, \mu \nu} \\
& =-\frac{1}{\sqrt{-\gamma}} \Lambda_{0}^{\mu}\left(H_{L \mu a} Z^{\prime a}+\Lambda_{1}^{\nu} H_{L \mu \nu}\right) .
\end{aligned}
$$

Let us write down the conserved quantities. By definition, the generalized momenta are

$$
P_{L} \equiv \frac{\partial \mathcal{L}^{P}}{\partial\left(\partial_{0} X^{L}\right)}=-T\left(\sqrt{-\gamma} \gamma^{0 n} g_{L N} \partial_{n} X^{N}-b_{L N} \partial_{1} X^{N}\right)
$$

For our ansatz (1.3), they take the form:

$$
P_{L}=-T \sqrt{-\gamma}\left[\left(\gamma^{01} g_{L b}-\frac{1}{\sqrt{-\gamma}} b_{L b}\right) Z^{\prime b}+\gamma^{0 n} \Lambda_{n}^{\nu} g_{L \nu}-\frac{1}{\sqrt{-\gamma}} \Lambda_{1}^{\nu} b_{L \nu}\right]
$$


The Lagrangian (2.11) does not depend on the coordinates $X^{\mu}$. Therefore, the conjugated momenta $P_{\mu}$ do not depend on the proper time $\tau^{4}$

$$
P_{\mu}(\sigma)=-T \sqrt{-\gamma}\left[\left(\gamma^{01} g_{\mu b}-\frac{1}{\sqrt{-\gamma}} b_{\mu b}\right) Z^{\prime b}+\gamma^{0 n} \Lambda_{n}^{\nu} g_{\mu \nu}-\frac{1}{\sqrt{-\gamma}} \Lambda_{1}^{\nu} b_{\mu \nu}\right], \partial_{0} P_{\mu}=0
$$

In order for our ansatz (1.3), (2.10) to be consistent with the action (2.1), the following conditions must be fulfilled

$$
\partial_{1} \mathcal{P}_{\mu} \equiv \frac{\partial \mathcal{P}_{\mu}}{\partial \sigma}=0
$$

where

$$
\begin{aligned}
& \mathcal{P}_{M} \equiv \frac{\partial \mathcal{L}^{P}}{\partial\left(\partial_{1} X^{M}\right)}=-T\left(\sqrt{-\gamma} \gamma^{1 n} g_{M N} \partial_{n} X^{N}+b_{M N} \partial_{0} X^{N}\right) \\
& =-T \sqrt{-\gamma}\left[\gamma^{11} g_{M b} Z^{\prime b}+\gamma^{1 n} \Lambda_{n}^{\nu} g_{M \nu}+\frac{1}{\sqrt{-\gamma}} \Lambda_{0}^{\nu} b_{M \nu}\right]
\end{aligned}
$$

This is because the equations of motion (2.2) can be rewritten as

$$
\frac{\partial P_{M}}{\partial \tau}+\frac{\partial \mathcal{P}_{M}}{\partial \sigma}-\frac{\partial \mathcal{L}^{P}}{\partial x^{M}}=0
$$

Hence, for the ansatz (1.3), (2.10), and for $M=\mu$, these equations take the form (2.17). Let us show this explicitly. In accordance with (2.10), the computation of $\Gamma_{\lambda, M N}$ and $H_{\lambda, M N}$ gives

$$
\begin{aligned}
\Gamma_{\lambda, a b} & =\frac{1}{2}\left(\partial_{a} g_{b \lambda}+\partial_{b} g_{a \lambda}\right), \quad \Gamma_{\lambda, \mu a}=\frac{1}{2} \partial_{a} g_{\mu \lambda}, \quad \Gamma_{\lambda, \mu \nu}=0 \\
H_{\lambda a b} & =\partial_{a} b_{b \lambda}+\partial_{b} b_{\lambda a}, \quad H_{\lambda \mu a}=\partial_{a} b_{\lambda \mu}, \quad H_{\lambda \mu \nu}=0
\end{aligned}
$$

Inserting these expressions in the part of the differential equations (2.15) corresponding to $L=\lambda$, and using the equalities $g_{M N}^{\prime}=Z^{\prime a} \partial_{a} g_{M N}, b_{M N}^{\prime}=Z^{\prime a} \partial_{a} b_{M N}$, one receives that the quantities

$$
\gamma^{11} g_{\lambda a}(\sigma) Z^{\prime a}(\sigma)+\gamma^{1 m} \Lambda_{m}^{\mu} g_{\lambda \mu}(\sigma)+\frac{1}{\sqrt{-\gamma}} \Lambda_{0}^{\mu} b_{\lambda \mu}(\sigma)
$$

do not depend on $\sigma$. Actually, they do not depend on $\tau$ too. Comparing (2.19) with (2.18), we see that they are connected with the constants of the motion $\mathcal{P}_{\mu}$ as

$$
\gamma^{11} g_{\mu a} Z^{\prime a}+\gamma^{1 n} \Lambda_{n}^{\nu} g_{\mu \nu}+\frac{1}{\sqrt{-\gamma}} \Lambda_{0}^{\nu} b_{\mu \nu}=-\frac{\mathcal{P}_{\mu}}{T \sqrt{-\gamma}}=\text { constants }
$$

From (2.14) and (2.20), one obtains the following compatibility condition

$$
\Lambda_{0}^{\nu} \mathcal{P}_{\nu}=0
$$

\footnotetext{
${ }^{4}$ Actually, all momenta $P_{M}$ do not depend on $\tau$, because there is no such dependence in (2.11).
} 
This equality may be interpreted as a solution of the constraint (2.14), which restricts the number of the independent parameters in the theory ${ }^{5}$.

With the help of (2.20), the other constraint, (2.13), can be rewritten in the form

$$
g_{a b} Z^{\prime a} Z^{\prime b}=\mathcal{U}
$$

where $\mathcal{U}$ is given by

$$
\mathcal{U}=\frac{1}{\gamma^{11}}\left[\gamma^{m n} \Lambda_{m}^{\mu} \Lambda_{n}^{\nu} g_{\mu \nu}+\frac{2 \Lambda_{1}^{\mu}}{T \sqrt{-\gamma}}\left(\mathcal{P}_{\mu}+T \Lambda_{0}^{\nu} b_{\mu \nu}\right)\right]
$$

Now, let us turn to the equations of motion (2.15), corresponding to $L=a$. In view of the conditions (2.10),

$$
\begin{aligned}
& \Gamma_{a, \mu b}=-\frac{1}{2}\left(\partial_{a} g_{b \mu}-\partial_{b} g_{a \mu}\right)=-\partial_{[a} g_{b] \mu}, \quad \Gamma_{a, \mu \nu}=-\frac{1}{2} \partial_{a} g_{\mu \nu}, \\
& H_{a \mu \nu}=\partial_{a} b_{\mu \nu} ; \quad H_{a b \nu}=\partial_{a} b_{b \nu}-\partial_{b} b_{a \nu}=2 \partial_{[a} b_{b] \nu} .
\end{aligned}
$$

By using this, one obtains

$$
g_{a b} Z^{\prime \prime b}+\Gamma_{a, b c} Z^{\prime b} Z^{\prime c}=\frac{1}{2} \partial_{a} \mathcal{U}+2 \partial_{[a} \mathcal{A}_{b]} Z^{\prime b}
$$

In (2.24), an effective scalar potential $\mathcal{U}$ and an effective 1-form gauge field $\mathcal{A}_{a}$ appeared. $\mathcal{U}$ is given in (2.23) (and is the same as in the effective constraint (2.22) ), and

$$
\mathcal{A}_{a}=\frac{1}{\gamma^{11}}\left(\gamma^{1 m} \Lambda_{m}^{\mu} g_{a \mu}+\frac{1}{\sqrt{-\gamma}} \Lambda_{0}^{\mu} b_{a \mu}\right) .
$$

Now our task is to find exact solutions of the nonlinear differential equations (2.22) and (2.24). How exactly this can be done is explained in Appendix A. Here, we give the final results only.

If the background seen by the string depends on only one coordinate $x^{a}$, the general solution for the string embedding coordinate $X^{a}(\tau, \sigma)=Z^{a}(\sigma)$ is given by

$$
\sigma\left(X^{a}\right)=\sigma_{0}+\int_{X_{0}^{a}}^{X^{a}}\left(\frac{\mathcal{U}}{g_{a a}}\right)^{-1 / 2} d x .
$$

When the background felt by the string depends on more than one coordinate $x^{a}$, the first integrals of the equations of motion for $Z^{a}(\sigma)=\left(Z^{r}, Z^{\alpha}\right)$, which also solve the constraint (2.22), are

$$
\begin{aligned}
& \left(g_{r r} Z^{\prime r}\right)^{2}=g_{r r}\left[\left(1-n_{\alpha}\right) \mathcal{U}-2 n_{\alpha}\left(\mathcal{A}_{r}-\partial_{r} f\right) Z^{\prime r}-\sum_{\alpha} \frac{D_{\alpha}\left(Z^{a \neq \alpha}\right)}{g_{\alpha \alpha}}\right]=F_{r}\left(Z^{r}\right) \geq 0, \\
& \left(g_{\alpha \alpha} Z^{\prime \alpha}\right)^{2}=D_{\alpha}\left(Z^{a \neq \alpha}\right)+g_{\alpha \alpha}\left[\mathcal{U}+2\left(\mathcal{A}_{r}-\partial_{r} f\right) Z^{\prime r}\right]=F_{\alpha}\left(Z^{\beta}\right) \geq 0,
\end{aligned}
$$

\footnotetext{
${ }^{5}$ When $\Lambda_{1}^{\mu}=0, g_{\mu a}=0$ and in diagonal worldsheet gauge $\left(\gamma^{01}=0\right)$, the constraint (2.14) and the condition (2.21) are identically satisfied. All classical string configurations belonging to the type (1.3), and the corresponding string theory backgrounds considered in [1] - [5], 17, [8], 10], 11], 14] - [20, [23] - 26, are particular cases of this particular case.
} 
where $Z^{r}$ is one of the coordinates $Z^{a}, Z^{\alpha}$ are the remaining ones, $n_{\alpha}$ is the number of $Z^{\alpha}$, and $D_{\alpha}, F_{a}$ are arbitrary functions of their arguments. The above expressions are valid, if the $g_{a b}$ part of the metric is diagonal one, and the following integrability conditions hold ${ }^{6}$

$$
\begin{aligned}
& \mathcal{A}_{a} \equiv\left(\mathcal{A}_{r}, \mathcal{A}_{\alpha}\right)=\left(\mathcal{A}_{r}, \partial_{\alpha} f\right), \quad \partial_{\alpha}\left(\frac{g_{\alpha \alpha}}{g_{a a}}\right)=0, \\
& \partial_{\alpha}\left(g_{r r} Z^{\prime r}\right)^{2}=0, \quad \partial_{r}\left(g_{\alpha \alpha} Z^{\prime \alpha}\right)^{2}=0 .
\end{aligned}
$$

\subsection{The tensionless limit}

The results obtained till now are not applicable to tensionless (null) strings, because the action (2.1) is proportional to the string tension $T$. The parameterization of the auxiliary worldsheet metric $\gamma^{m n}$, which is appropriate for considering the zero tension $\operatorname{limit} T \rightarrow 0$, is the following 32,33 :

$$
\gamma^{00}=-1, \quad \gamma^{01}=\lambda^{1}, \quad \gamma^{11}=\left(2 \lambda^{0} T\right)^{2}-\left(\lambda^{1}\right)^{2}, \quad \operatorname{det}\left(\gamma^{m n}\right)=-\left(2 \lambda^{0} T\right)^{2}
$$

Now, the action (2.1) becomes

$$
S_{\lambda}=\int d^{2} \xi\left\{\frac{1}{4 \lambda^{0}}\left[G_{00}-2 \lambda^{1} G_{01}+\left(\lambda^{1}\right)^{2} G_{11}-\left(2 \lambda^{0} T\right)^{2} G_{11}\right]+T B_{01}\right\} .
$$

Here, $\lambda^{n}$ are the Lagrange multipliers, whose equations of motion generate the independent constraints.

In these notations, the constraints (2.13) and (2.14), the equations of motion (2.15), and the conserved quantities (2.16), (2.20) take the form

$$
\begin{gathered}
g_{a b} Z^{\prime a} Z^{\prime b}+2 \Lambda_{1}^{\mu} g_{\mu b} Z^{\prime b}+\left[\frac{\Lambda_{0}^{\mu} \Lambda_{0}^{\nu}}{\left(2 \lambda^{0} T\right)^{2}-\left(\lambda^{1}\right)^{2}}+\Lambda_{1}^{\mu} \Lambda_{1}^{\nu}\right] g_{\mu \nu}=0 \\
\Lambda_{0}^{\mu}\left\{g_{\mu a} Z^{\prime a}+\left[\frac{\lambda^{1} \Lambda_{0}^{\nu}}{\left(2 \lambda^{0} T\right)^{2}-\left(\lambda^{1}\right)^{2}}+\Lambda_{1}^{\nu}\right] g_{\mu \nu}\right\}=0 \\
g_{L b} Z^{\prime \prime b}+\Gamma_{L, b c} Z^{\prime b} Z^{\prime c}+2\left[\frac{\lambda^{1} \Lambda_{0}^{\mu}}{\left(2 \lambda^{0} T\right)^{2}-\left(\lambda^{1}\right)^{2}}+\Lambda_{1}^{\mu}\right] \Gamma_{L, \mu b} Z^{\prime b} \\
+\left[\frac{\Lambda_{0}^{\mu}\left(2 \lambda^{1} \Lambda_{1}^{\nu}-\Lambda_{0}^{\nu}\right)}{\left(2 \lambda^{0} T\right)^{2}-\left(\lambda^{1}\right)^{2}}+\Lambda_{1}^{\mu} \Lambda_{1}^{\nu}\right] \Gamma_{L, \mu \nu}=-\frac{1}{\left(2 \lambda^{0} T\right)^{2}-\left(\lambda^{1}\right)^{2}} \Lambda_{0}^{\mu}\left(H_{L \mu a} Z^{\prime a}+\Lambda_{1}^{\nu} H_{L \mu \nu}\right) . \\
P_{\mu}(\sigma)=\frac{1}{2 \lambda^{0}}\left[\left(-\lambda^{1} g_{\mu a}+2 \lambda^{0} T b_{\mu a}\right) Z^{\prime a}+\left(\Lambda_{0}^{\nu}-\lambda^{1} \Lambda_{1}^{\nu}\right) g_{\mu \nu}+2 \lambda^{0} T \Lambda_{1}^{\nu} b_{\mu \nu}\right], \\
\mathcal{P}_{\mu}=-\frac{1}{2 \lambda^{0}}\left\{\left[\left(2 \lambda^{0} T\right)^{2}-\left(\lambda^{1}\right)^{2}\right]\left(g_{\mu a} Z^{\prime a}+\Lambda_{1}^{\nu} g_{\mu \nu}\right)+\Lambda_{0}^{\nu}\left(\lambda^{1} g_{\mu \nu}+2 \lambda^{0} T b_{\mu \nu}\right)\right\} .
\end{gathered}
$$

\footnotetext{
${ }^{6}$ In all cases, considered in [1] - 27, $\mathcal{A}_{a} \equiv 0$.
} 
The reduced equations of motion and constraint (2.24) and (2.22) have the same form, but now, the effective potential (2.23) and the effective gauge field (2.25) are given by

$$
\begin{aligned}
& \mathcal{U}=\left[\frac{\Lambda_{0}^{\mu}\left(2 \lambda^{1} \Lambda_{1}^{\nu}-\Lambda_{0}^{\nu}\right)}{\left(2 \lambda^{0} T\right)^{2}-\left(\lambda^{1}\right)^{2}}+\Lambda_{1}^{\mu} \Lambda_{1}^{\nu}\right] g_{\mu \nu}+\frac{4 \lambda^{0}}{\left(2 \lambda^{0} T\right)^{2}-\left(\lambda^{1}\right)^{2}} \Lambda_{1}^{\mu}\left(\mathcal{P}_{\mu}+T \Lambda_{0}^{\nu} b_{\mu \nu}\right), \\
& \mathcal{A}_{a}=\left[\frac{\lambda^{1} \Lambda_{0}^{\nu}}{\left(2 \lambda^{0} T\right)^{2}-\left(\lambda^{1}\right)^{2}}+\Lambda_{1}^{\nu}\right] g_{a \nu}+\frac{2 \lambda^{0} T}{\left(2 \lambda^{0} T\right)^{2}-\left(\lambda^{1}\right)^{2}} \Lambda_{0}^{\mu} b_{a \mu} .
\end{aligned}
$$

If one sets $\lambda^{1}=0$ and $2 \lambda^{0} T=1$, this will correspond to conformal gauge, as it should be. If one puts $T=0$ in the above formulas, they will describe tensionless strings.

\section{$3 \quad$ Exact solutions for more general string embedding}

In this section, we will use the ansatz (1.4) for the string coordinates, which corresponds to more general string embedding. Here, compared with (1.3), $X^{\mu}$ are allowed to vary non-linearly with the worldsheet spatial parameter $\sigma$. Of course, the conditions (2.10) on the background fields are also fulfilled.

Taking into account the ansatz (1.4), one obtains that the induced fields $G_{m n}$ and $B_{m n}$, the Lagrangian density, the constraints (2.8) and (2.7) respectively, and the EulerLagrange equations for $X^{M}(2.9)$ are given by

$$
\begin{gathered}
G_{00}=\Lambda_{0}^{\mu} \Lambda_{0}^{\nu} g_{\mu \nu}, \quad G_{01}=\Lambda_{0}^{\mu}\left(g_{\mu N} Z^{\prime N}+\Lambda_{1}^{\nu} g_{\mu \nu}\right) \\
G_{11}=g_{M N} Z^{\prime M} Z^{\prime N}+2 \Lambda_{1}^{\mu} g_{\mu N} Z^{\prime N}+\Lambda_{1}^{\mu} \Lambda_{1}^{\nu} g_{\mu \nu} \\
B_{01}=\Lambda_{0}^{\mu}\left(b_{\mu N} Z^{\prime N}+\Lambda_{1}^{\nu} b_{\mu \nu}\right) ; \\
\mathcal{L}^{G A}(\sigma)=-\frac{T}{2} \sqrt{-\gamma}\left[\gamma^{11} g_{M N} Z^{\prime M} Z^{\prime N}+2\left(\gamma^{1 m} \Lambda_{m}^{\mu} g_{\mu N}-\frac{\Lambda_{0}^{\mu} b_{\mu N}}{\sqrt{-\gamma}}\right) Z^{\prime N}+\right. \\
\left.+\gamma^{m n} \Lambda_{m}^{\mu} \Lambda_{n}^{\nu} g_{\mu \nu}-\frac{2 \Lambda_{0}^{\mu} \Lambda_{1}^{\nu} b_{\mu \nu}}{\sqrt{-\gamma}}\right] \\
\gamma^{11} g_{M N} Z^{\prime M} Z^{\prime N}+2 \gamma^{11} \Lambda_{1}^{\mu} g_{\mu N} Z^{\prime N}-\left(\gamma^{00} \Lambda_{0}^{\mu} \Lambda_{0}^{\nu}-\gamma^{11} \Lambda_{1}^{\mu} \Lambda_{1}^{\nu}\right) g_{\mu \nu}=0 \\
\Lambda_{0}^{\mu}\left(\gamma^{11} g_{\mu N} Z^{\prime N}+\gamma^{1 n} \Lambda_{n}^{\nu} g_{\mu \nu}\right)=0 ; \\
\gamma^{11}\left(g_{L N} Z^{\prime \prime N}+\Gamma_{L, M N} Z^{\prime M} Z^{\prime N}\right)+2 \gamma^{1 m} \Lambda_{m}^{\mu} \Gamma_{L, \mu N} Z^{\prime N}+\gamma^{m n} \Lambda_{m}^{\mu} \Lambda_{n}^{\nu} \Gamma_{L, \mu \nu}= \\
=-\frac{1}{\sqrt{-\gamma}} \Lambda_{0}^{\mu}\left(H_{L \mu N} Z^{\prime N}+\Lambda_{1}^{\nu} H_{L \mu \nu}\right)
\end{gathered}
$$

The quantities $P_{L}, \mathcal{P}_{L}$ can be found as before, and now they are

$$
\begin{aligned}
& \left(\gamma^{01} g_{L N}-\frac{b_{L N}}{\sqrt{-\gamma}}\right) Z^{\prime N}+\gamma^{0 n} \Lambda_{n}^{\nu} g_{L \nu}-\frac{\Lambda_{1}^{\nu} b_{L \nu}}{\sqrt{-\gamma}}=-\frac{P_{L}}{T \sqrt{-\gamma}}, \quad \partial_{0} P_{L}=0 \\
& \gamma^{11} g_{L N} Z^{\prime N}+\gamma^{1 n} \Lambda_{n}^{\nu} g_{L \nu}+\frac{\Lambda_{0}^{\nu} b_{L \nu}}{\sqrt{-\gamma}}=-\frac{\mathcal{P}_{L}}{T \sqrt{-\gamma}}, \quad \partial_{0} \mathcal{P}_{L}=0, \quad \partial_{1} \mathcal{P}_{\mu}=0
\end{aligned}
$$


The compatibility condition following from the constraint (3.3) and from (3.6) coincides with the previous one (2.21).

As in the previous section, the equations (3.4) for $L=\lambda$ lead to $\partial_{1} \mathcal{P}_{\lambda}=0$. Consequently, our next task is to consider the equations (3.4) for $L=a$ and the constraint (3.2). First of all, we will eliminate the variables $Z^{\prime \mu}$ from them. To this end, we express $Z^{\prime \mu}$ through $Z^{\prime a}$ by using (3.6):

$$
Z^{\prime \mu}=-\frac{\gamma^{1 m}}{\gamma^{11}} \Lambda_{m}^{\mu}-\left(g^{-1}\right)^{\mu \nu}\left[g_{\nu a} Z^{\prime a}+\frac{1}{T \sqrt{-\gamma} \gamma^{11}}\left(\mathcal{P}_{\nu}+T \Lambda_{0}^{\rho} b_{\nu \rho}\right)\right]
$$

With the help of (3.7) and (2.21), the equations (3.4) for $L=a$ and the constraint (3.2) acquire the form

$$
\begin{aligned}
& h_{a b} Z^{\prime \prime b}+\Gamma_{a, b c}^{\mathbf{h}} Z^{\prime b} Z^{\prime c}=\frac{1}{2} \partial_{a} \mathcal{U}^{\mathbf{h}}+2 \partial_{[a} \mathcal{A}_{b]}^{\mathbf{h}} Z^{\prime b}, \\
& h_{a b} Z^{\prime a} Z^{\prime b}=\mathcal{U}^{\mathbf{h}},
\end{aligned}
$$

where a new, effective metric appeared

$$
h_{a b}=g_{a b}-g_{a \mu}\left(g^{-1}\right)^{\mu \nu} g_{\nu b} .
$$

$\Gamma_{a, b c}^{\mathbf{h}}$ is the symmetric connection corresponding to this metric

$$
\Gamma_{a, b c}^{\mathbf{h}}=\frac{1}{2}\left(\partial_{b} h_{c a}+\partial_{c} h_{b a}-\partial_{a} h_{b c}\right) .
$$

The effective scalar and gauge potentials, expressed through the background fields, are as follows

$$
\begin{aligned}
\mathcal{U}^{\mathbf{h}} & =\frac{1}{\gamma\left(\gamma^{11}\right)^{2}}\left[\Lambda_{0}^{\mu} \Lambda_{0}^{\nu} g_{\mu \nu}+\frac{1}{T^{2}}\left(\mathcal{P}_{\mu}+T \Lambda_{0}^{\rho} b_{\mu \rho}\right)\left(g^{-1}\right)^{\mu \nu}\left(\mathcal{P}_{\nu}+T \Lambda_{0}^{\lambda} b_{\nu \lambda}\right)\right], \\
\mathcal{A}_{a}^{\mathbf{h}} & =-\frac{1}{T \sqrt{-\gamma} \gamma^{11}}\left[g_{a \mu}\left(g^{-1}\right)^{\mu \nu}\left(\mathcal{P}_{\nu}+T \Lambda_{0}^{\rho} b_{\nu \rho}\right)-T \Lambda_{0}^{\rho} b_{a \rho}\right] .
\end{aligned}
$$

We point out the qualitatively different behavior of the potentials $\mathcal{U}^{\mathbf{h}}$ and $\mathcal{A}_{a}^{\mathbf{h}}$, compared to $\mathcal{U}$ and $\mathcal{A}_{a}$ from the previous section, due to the appearance of the inverse metric $\left(g^{-1}\right)^{\mu \nu}$ in the above expressions.

Since the equations (2.24), (2.22) and (3.8), (3.9) have the same form, for obtaining exact string solutions, we can proceed as before and use the derived formulas after the replacements $(g, \Gamma, \mathcal{U}, \mathcal{A}) \rightarrow\left(h, \Gamma^{\mathbf{h}}, \mathcal{U}^{\mathbf{h}}, \mathcal{A}^{\mathbf{h}}\right)$ (see Appendix A). In particular, the solution depending on one of the coordinates $X^{a}$ will be

$$
\sigma\left(X^{a}\right)=\sigma_{0}+\int_{X_{0}^{a}}^{X^{a}} d x\left(\frac{\mathcal{U}^{\mathbf{h}}}{h_{a a}}\right)^{-1 / 2} .
$$

In this case by integrating (3.7), and replacing the solution for $Z^{\mu}$ in the ansatz (1.4), one obtains solution for the string coordinates $X^{\mu}$ of the type $X^{\mu}\left(\tau, X^{a}\right)$ :

$$
\begin{aligned}
& X^{\mu}\left(\tau, X^{a}\right)=X_{0}^{\mu}+\Lambda_{0}^{\mu}\left[\tau-\frac{\gamma^{01}}{\gamma^{11}} \sigma\left(X^{a}\right)\right]- \\
& -\int_{X_{0}^{a}}^{X^{a}}\left(g^{-1}\right)^{\mu \nu}\left[g_{\nu a}+\frac{\left(\mathcal{P}_{\nu}+T \Lambda_{0}^{\rho} b_{\nu \rho}\right)}{T \sqrt{-\gamma} \gamma^{11}}\left(\frac{\mathcal{U}^{\mathrm{h}}}{h_{a a}}\right)^{-1 / 2}\right] d x .
\end{aligned}
$$


To write down a solution of the type $X^{\mu}(\tau, \sigma)$, one have to invert the solution (3.10): $\sigma\left(X^{a}\right) \rightarrow X^{a}(\sigma)$. Then, $X^{\mu}(\tau, \sigma)$ are given by

$$
\begin{aligned}
& X^{\mu}(\tau, \sigma)=X_{0}^{\mu}+\Lambda_{0}^{\mu}\left(\tau-\frac{\gamma^{01}}{\gamma^{11}} \sigma\right)- \\
& -\int_{\sigma_{0}}^{\sigma}\left(g^{-1}\right)^{\mu \nu}\left[\frac{\left(\mathcal{P}_{\nu}+T \Lambda_{0}^{\rho} b_{\nu \rho}\right)}{T \sqrt{-\gamma} \gamma^{11}}+g_{\nu a}\left(\frac{\mathcal{U}^{\mathrm{h}}}{h_{a a}}\right)^{1 / 2}\right] d \sigma .
\end{aligned}
$$

Let us also give the expression for $P_{\mu}$ after the elimination of $Z^{\prime \mu}$ from (3.5)

$$
\begin{aligned}
& P_{\mu}(\sigma)=T\left[b_{\mu a}-b_{\mu \nu}\left(g^{-1}\right)^{\nu \lambda} g_{\lambda a}\right] Z^{\prime a} \\
& +\frac{1}{\gamma^{11}}\left\{\gamma^{01} \mathcal{P}_{\mu}+\frac{1}{\sqrt{-\gamma}}\left[T \Lambda_{0}^{\nu} g_{\mu \nu}-b_{\mu \nu}\left(g^{-1}\right)^{\nu \lambda}\left(\mathcal{P}_{\lambda}+T \Lambda_{0}^{\rho} b_{\lambda \rho}\right)\right]\right\} .
\end{aligned}
$$

These equalities connect the conserved momenta $P_{\mu}$ with the constants of the motion $\mathcal{P}_{\mu}$.

To be able to take the tensionless limit $T \rightarrow 0$ in the above formulas, we must use the $\lambda$-parameterization (2.26) of $\gamma^{m n}$. The quantities, which depend on this parameterization, and appear in the reduced equations of motion and constraint (3.8), (3.9), and therefore - in the solutions, are $\mathcal{U}^{\mathbf{h}}$ and $\mathcal{A}_{a}^{\mathbf{h}}$. Now, they read

$$
\begin{aligned}
\mathcal{U}^{\mathbf{h}} & =-\frac{\left(2 \lambda^{0}\right)^{2}}{\left[\left(2 \lambda^{0} T\right)^{2}-\left(\lambda^{1}\right)^{2}\right]^{2}}\left[T^{2} \Lambda_{0}^{\mu} \Lambda_{0}^{\nu} g_{\mu \nu}+\left(\mathcal{P}_{\mu}+T \Lambda_{0}^{\rho} b_{\mu \rho}\right)\left(g^{-1}\right)^{\mu \nu}\left(\mathcal{P}_{\nu}+T \Lambda_{0}^{\lambda} b_{\nu \lambda}\right)\right] \\
\mathcal{A}_{a}^{\mathbf{h}} & =-\frac{2 \lambda^{0}}{\left(2 \lambda^{0} T\right)^{2}-\left(\lambda^{1}\right)^{2}}\left[g_{a \mu}\left(g^{-1}\right)^{\mu \nu}\left(\mathcal{P}_{\nu}+T \Lambda_{0}^{\rho} b_{\nu \rho}\right)-T \Lambda_{0}^{\rho} b_{a \rho}\right]
\end{aligned}
$$

If one sets $\lambda^{1}=0$ and $2 \lambda^{0} T=1$, the conformal gauge results are obtained. If one puts $T=0$ in the above equalities, they will correspond to tensionless strings. For instance, the solution $X^{\mu}\left(\tau, X^{a}\right)$ reduces to

$$
\begin{aligned}
& X^{\mu}\left(\tau, X^{a}\right)_{T=0}=X_{0}^{\mu}+\Lambda_{0}^{\mu}\left[\tau+\frac{\sigma\left(X^{a}\right)}{\lambda^{1}}\right]- \\
& -\int_{X_{0}^{a}}^{X^{a}}\left(g^{-1}\right)^{\mu \nu}\left[g_{\nu a}-\frac{2 \lambda^{0}}{\left(\lambda^{1}\right)^{2}} \mathcal{P}_{\nu}\left(\frac{\mathcal{U}^{\mathrm{h}}}{h_{a a}}\right)_{T=0}^{-1 / 2}\right] d x .
\end{aligned}
$$

\section{Examples}

In the previous two sections, we described a general approach for solving the string equations of motion and constraints in the background fields $g_{M N}(x)$ and $b_{M N}(x)$, based on the ansatzes (1.3) and (1.4). In this section, as an illustration of the previously obtained generic results, we will establish the correspondence with the particular cases considered in [1] - in the framework of the linear ansatz (1.3), and in [13] - in the framework of the nonlinear ansatz (1.4). After that, we will find new exact string solutions in $A d S_{5}$ black hole and in $A d S_{5} \times S^{5}$ backgrounds. 


\subsection{Comparing with known solutions}

In [1, the string theory background is $A d S_{5} \times S^{5}$, with field theory dual $\mathcal{N}=4 S U(N)$ $S Y M$ in four dimensional flat space-time. The metric of the $A d S_{5}$ is taken in global coordinates, so that the string energy is identified with the conformal dimension in the dual CFT

$$
\begin{aligned}
& d s_{A d S_{5}}^{2}=R^{2}\left(-\cosh ^{2} \rho d t^{2}+d \rho^{2}+\sinh ^{2} \rho d \Omega_{3}^{2}\right), \\
& d \Omega_{3}^{2}=d \theta^{2}+\cos ^{2} \theta\left(d \psi^{2}+\cos ^{2} \psi d \phi^{2}\right), \quad R^{4}=\lambda \alpha^{\prime 2} .
\end{aligned}
$$

As our first example, we will consider one of the classical string configurations analyzed in [1]. Namely, we will consider a closed spinning string in $A d S_{5}$ given by the following ansatz

$$
t=e \tau, \quad \rho=\rho(\sigma), \quad \phi=e \omega \tau, \quad e, \omega=\text { constants }
$$

Therefore, the background seen by the string is

$$
d s^{2}=R^{2}\left(-\cosh ^{2} \rho d t^{2}+d \rho^{2}+\sinh ^{2} \rho d \phi^{2}\right), \quad b_{M N}=0 .
$$

This metric does not depend on $x^{0}=t$ and $x^{2}=\phi$, i.e. $X^{\mu}=X^{0,2}$ and $X^{a}=X^{1}=\rho(\sigma)$ in our notation. Comparing the ansatzes (1.3) and (4.1), one sees that the latter is particular case of the former, corresponding to

$$
\Lambda_{0}^{0}=e, \quad \Lambda_{1}^{0}=\Lambda_{1}^{2}=0, \quad \Lambda_{0}^{2}=e \omega .
$$

The conserved momenta $P_{\mu}$, obtained from (2.16), are

$$
P_{0}=T \sqrt{-\gamma} \gamma^{00} e R^{2} \cosh ^{2} \rho, \quad P_{2}=-T \sqrt{-\gamma} \gamma^{00} e \omega R^{2} \sinh ^{2} \rho .
$$

So, the energy and the spin are given by

$$
\begin{aligned}
& E=-\int_{0}^{2 \pi} d \sigma P_{0}=-T \sqrt{-\gamma} \gamma^{00} e R^{2} \int_{0}^{2 \pi} d \sigma \cosh ^{2} \rho, \\
& S=\int_{0}^{2 \pi} d \sigma P_{2}=-T \sqrt{-\gamma} \gamma^{00} e \omega R^{2} \int_{0}^{2 \pi} d \sigma \sinh ^{2} \rho .
\end{aligned}
$$

In diagonal worldsheet gauge, $\gamma^{01}=0$, the first integrals (2.20) are identically zero: $\mathcal{P}_{\mu} \equiv 0, \mu=0,2$. In this gauge, the constraint (2.14) and the compatibility condition (2.21) are also identically satisfied. Thus, it remains to solve the equations of motion (2.24) and the constraint (2.22). The background (4.2) depends on only one coordinate $x^{1}=\rho$. We know from our considerations in Appendix A that in this case, the effective constraint (2.22) is first integral of the equation (2.24). It reads

$$
\rho^{\prime 2}=-\frac{\gamma^{00}}{\gamma^{11}} e^{2}\left(\cosh ^{2} \rho-\omega^{2} \sinh ^{2} \rho\right) .
$$

It follows from here that

$$
d \sigma=\frac{d \rho}{e \sqrt{-\frac{\gamma^{00}}{\gamma^{11}}\left(\cosh ^{2} \rho-\omega^{2} \sinh ^{2} \rho\right)}} .
$$


By integrating this equality, one obtains the string solution $\sigma(\rho)$, given in the general case by (A.4).

In conformal gauge, (4.3), (4.4), (4.5) and (4.6) reduce to the corresponding formulas derived in [1]. In the tensionless limit, after using the $\lambda$-parameterization (2.26) of $\gamma^{m n}$, (4.3), (4.4) and (4.6) take the form

$$
\begin{aligned}
& E_{T=0}=\frac{e R^{2}}{2 \lambda^{0}} \int_{0}^{2 \pi} d \sigma \cosh ^{2} \rho \\
& S_{T=0}=\frac{e \omega R^{2}}{2 \lambda^{0}} \int_{0}^{2 \pi} d \sigma \sinh ^{2} \rho \\
& d \sigma_{T=0}=\frac{\lambda^{1} d \rho}{e \sqrt{\left(\omega^{2} \sinh ^{2} \rho-\cosh ^{2} \rho\right)}} .
\end{aligned}
$$

In [13, the string theory background is $A d S_{3} \times S^{3} \times \mathcal{M}$, with NS-NS 2-form gauge field. In accordance with the $A d S / C F T$ duality, the string theory on $A d S_{3} \times S^{3} \times \mathcal{M}$ is dual to a superconformal field theory on a cylinder, which is the boundary of $A d S_{3}$ in global coordinates. In such coordinates, the NS-NS $A d S_{3}$ background can be written as

$$
d s_{A d S_{3}}^{2}=R^{2}\left[-\left(1+r^{2}\right) d t^{2}+\frac{d r^{2}}{1+r^{2}}+r^{2} d \phi^{2}\right], \quad b_{t \phi}=R^{2} r^{2} .
$$

As our second example, we will consider the first of the two classical string configurations discussed in 13

$$
t=c_{1} \tau+\tilde{t}(\sigma), \quad r=r(\sigma), \quad \phi=c_{2} \tau+\tilde{\phi}(\sigma) .
$$

The above background does not depend on $x^{0}=t$ and $x^{2}=\phi$. Therefore, $\mu=(0,2)$, $a=1$, and comparing (4.8) with (1.4), one obtains

$$
\begin{aligned}
& \Lambda_{0}^{0}=c_{1}, \quad \Lambda_{0}^{2}=c_{2}, \quad \Lambda_{1}^{\mu}=0, \\
& Z^{0}(\sigma)=\tilde{t}(\sigma), \quad Z^{1}(\sigma)=r(\sigma), \quad Z^{2}(\sigma)=\tilde{\phi}(\sigma) .
\end{aligned}
$$

The action (2.1) for this string embedding, in worldsheet gauge $\gamma^{m n}=$ constants, is

$$
\begin{aligned}
& S^{P}=-\frac{T R^{2}}{2} \sqrt{-\gamma} \int d \tau d \sigma\left[-\left(1+r^{2}\right)\left(\gamma^{00} c_{1}^{2}+2 \gamma^{01} c_{1} \tilde{t}^{\prime}+\gamma^{11} \tilde{t}^{\prime 2}\right)\right. \\
& \left.+\gamma^{11} \frac{r^{\prime 2}}{1+r^{2}}+r^{2}\left(\gamma^{00} c_{2}^{2}+2 \gamma^{01} c_{2} \tilde{\phi}^{\prime}+\gamma^{11} \tilde{\phi}^{\prime 2}\right)-\frac{2}{\sqrt{-\gamma}} r^{2}\left(c_{1} \tilde{\phi}^{\prime}-c_{2} \tilde{t}^{\prime}\right)\right] .
\end{aligned}
$$

The explicit expressions for the conserved momenta $P_{\mu}$ can be obtained from (3.5). By using them, one is able to compute the string energy and spin:

$$
\begin{aligned}
& E=-\int_{0}^{2 \pi} d \sigma P_{0}=-T \sqrt{-\gamma} R^{2} \int_{0}^{2 \pi} d \sigma\left[\left(\gamma^{00} c_{1}+\gamma^{01} t^{\prime}\right)\left(1+r^{2}\right)+\frac{r^{2} \phi^{\prime}}{\sqrt{-\gamma}}\right] \\
& S=\int_{0}^{2 \pi} d \sigma P_{2}=-T \sqrt{-\gamma} R^{2} \int_{0}^{2 \pi} d \sigma\left(\gamma^{00} c_{2}+\gamma^{01} \phi^{\prime}+\frac{t^{\prime}}{\sqrt{-\gamma}}\right) r^{2}
\end{aligned}
$$


As we know from Sec.3, the quantities $\mathcal{P}_{\mu}$, given in (3.6), are first integrals for this part of the equations of motion (3.4), which correspond to $L=\mu$. For the case under consideration, (3.6) leads to the following expressions for $\mathcal{P}_{\mu}$ :

$$
\begin{aligned}
& \mathcal{P}_{0}=T \sqrt{-\gamma} R^{2}\left[\left(\gamma^{11} \tilde{t}^{\prime}+\gamma^{01} c_{1}\right)\left(1+r^{2}\right)-\frac{c_{2} r^{2}}{\sqrt{-\gamma}}\right]=\text { constant }, \\
& \mathcal{P}_{2}=-T \sqrt{-\gamma} R^{2}\left(\gamma^{11} \tilde{\phi}^{\prime}+\gamma^{01} c_{2}-\frac{c_{1}}{\sqrt{-\gamma}}\right) r^{2}=\text { constant } .
\end{aligned}
$$

The above equalities are first order ordinary differential equations for $\tilde{t}(\sigma)$ and $\tilde{\phi}(\sigma)$, which rewritten in normal form read

$$
\tilde{t}^{\prime}=\frac{c_{2} r^{2}-k_{1}}{\sqrt{-\gamma} \gamma^{11}\left(1+r^{2}\right)}-\frac{\gamma^{01}}{\gamma^{11}} c_{1}, \quad \tilde{\phi}^{\prime}=\frac{c_{1} r^{2}-k_{2}}{\sqrt{-\gamma} \gamma^{11} r^{2}}-\frac{\gamma^{01}}{\gamma^{11}} c_{2},
$$

where

$$
k_{1} \equiv-\frac{\mathcal{P}_{0}}{T R^{2}}, \quad k_{2} \equiv \frac{\mathcal{P}_{2}}{T R^{2}}
$$

The equations (4.12) can be solved, if the function $r=r(\sigma)$ is already known. Before explaining how to find it, let us consider the compatibility condition (2.21), which is the same for both ansatzes - (1.3) and (1.4). In our case, it gives

$$
c_{1} k_{1}=c_{2} k_{2}
$$

from where one can eliminate one of the free parameters in the theory. From now on, we will use that $c_{2}=c_{1} k_{1} / k_{2}$.

Now, we are going to solve the equations of motion (3.8) and the constraint (3.9). The background fields in (4.7) depend on only one coordinate $x^{1}=r$. Therefore, as we already know, the effective constraint (3.9) is first integral of the equation of motion (3.8) for this coordinate.Thus, we have to only solve the constraint ([3.9), which in the case under consideration reads

$$
r^{\prime 2}=-\frac{\alpha \beta}{\gamma\left(\gamma^{11}\right)^{2} k_{2}^{2} r^{2}}\left(\frac{k_{2}^{2}}{\alpha}-r^{2}\right)\left(r^{2}-\frac{k_{2}^{2}}{\beta}\right),
$$

where

$$
\alpha=k_{1}^{2}-k_{2}^{2}, \quad \beta=c_{1}\left(c_{1}+2 k_{2}\right) .
$$

To find solution of this equation, we apply our general formula (3.10) and obtain $\left(\sigma_{0}=0\right)$ :

$$
\begin{aligned}
& \sigma(r)=\frac{\sqrt{-\gamma} \gamma^{11} k_{2}}{2 \sqrt{\alpha \beta}} \int \frac{d r^{2}}{\sqrt{\left(\frac{k_{2}^{2}}{\alpha}-r^{2}\right)\left(r^{2}-\frac{k_{2}^{2}}{\beta}\right)}} \\
& =-\sqrt{-\gamma} \gamma^{11} \frac{k_{2}}{2 \sqrt{\alpha \beta}} \arcsin \left[\frac{\frac{2 \alpha \beta}{k_{2}^{2}} r^{2}-(\alpha+\beta)}{\alpha-\beta}\right] .
\end{aligned}
$$


By inverting this solution, one receives

$$
r^{2}(\sigma)=\frac{k_{2}^{2}}{2 \alpha \beta}\left[(\alpha+\beta)-(\alpha-\beta) \sin \left(\frac{2 \sqrt{\alpha \beta}}{\sqrt{-\gamma} \gamma^{11} k_{2}} \sigma\right)\right] .
$$

Now, we can write down the solutions $X^{\mu}=X^{\mu}(\tau, r)$ for the remaining string coordinates, by using (3.11):

$$
\begin{aligned}
& X^{0}(\tau, r) \equiv t(\tau, r)=c_{1} \tau+\frac{c_{1}}{\gamma^{11}}\left(\frac{k_{1}}{\sqrt{-\gamma} k_{2}}-\gamma^{01}\right) \sigma(r) \\
& -\frac{k_{1}\left(c_{1}+k_{2}\right)}{2 \sqrt{\alpha \beta}} \int \frac{d r^{2}}{\left(r^{2}+1\right) \sqrt{\left(\frac{k_{2}^{2}}{\alpha}-r^{2}\right)\left(r^{2}-\frac{k_{2}^{2}}{\beta}\right)}}, \\
& X^{2}(\tau, r) \equiv \phi(\tau, r)=c_{1} \frac{k_{1}}{k_{2}} \tau+\frac{c_{1}}{\gamma^{11}}\left(\frac{1}{\sqrt{-\gamma}}-\gamma^{01} \frac{k_{1}}{k_{2}}\right) \sigma(r) \\
& -\frac{k_{2}^{2}}{2 \sqrt{\alpha \beta}} \int \frac{d r^{2}}{r^{2} \sqrt{\left(\frac{k_{2}^{2}}{\alpha}-r^{2}\right)\left(r^{2}-\frac{k_{2}^{2}}{\beta}\right)}} .
\end{aligned}
$$

Let us also give the connection between the conserved momenta $P_{\mu}$ and the constants of the motion $\mathcal{P}_{\mu}$ following from (3.13):

$$
\begin{aligned}
& P_{0}=\frac{1}{\gamma^{11}}\left[\gamma^{01} \mathcal{P}_{0}-\frac{1}{\sqrt{-\gamma}}\left(\mathcal{P}_{2}+c_{1} T R^{2}\right)\right], \\
& P_{2}=\frac{1}{\gamma^{11}}\left[\gamma^{01} \mathcal{P}_{2}-\frac{1}{\sqrt{-\gamma}}\left(\mathcal{P}_{0}-\frac{\mathcal{P}_{0}+c_{2} T R^{2} r^{2}}{r^{2}+1}\right)\right] .
\end{aligned}
$$

By integrating these equalities, one obtains the following expressions for the string energy (4.10) and spin (4.11)

$$
\begin{aligned}
& E=-\frac{2 \pi}{\gamma^{11}}\left[\gamma^{01} \mathcal{P}_{0}-\frac{\mathcal{P}_{2}}{\sqrt{-\gamma}}\left(1+c_{1} \frac{T R^{2}}{\mathcal{P}_{2}}\right)\right], \\
& S=\frac{2 \pi}{\gamma^{11}}\left[\gamma^{01} \mathcal{P}_{2}-\frac{\mathcal{P}_{0}}{\sqrt{-\gamma}}\left(1+c_{1} \frac{T R^{2}}{\mathcal{P}_{2}}\right)\left(1-\frac{1}{2 \pi} \int_{0}^{2 \pi} \frac{d \sigma}{r^{2}(\sigma)+1}\right)\right] .
\end{aligned}
$$

In the particular case of conformal gauge, (4.9) - (4.17) reproduce the corresponding results derived in [13]. To write down the solutions for $X^{\mu}$ as functions of $\tau$ and $\sigma$ (instead of $\tau$ and $r$ ), as given in [13, it is enough for one to replace (4.15) into (3.12).

In the tensionless limit $T \rightarrow 0$, after using the $\lambda$-parameterization (2.26) of $\gamma^{m n}$, one obtains the following exact string solutions

$$
\begin{aligned}
& \sigma(r)_{T=0}=\frac{\left(\lambda^{1} R\right)^{2}}{4 \lambda^{0}} \int \frac{d r^{2}}{\sqrt{\left(\mathcal{P}_{0}^{2}-\mathcal{P}_{2}^{2}\right) r^{2}-\mathcal{P}_{2}^{2}}}=\frac{\left(\lambda^{1} R\right)^{2}}{2 \lambda^{0}\left(\mathcal{P}_{0}^{2}-\mathcal{P}_{2}^{2}\right)} \sqrt{\left(\mathcal{P}_{0}^{2}-\mathcal{P}_{2}^{2}\right) r^{2}-\mathcal{P}_{2}^{2}}, \\
& r^{2}(\sigma)_{T=0}=\frac{\left(2 \lambda^{0}\right)^{2}\left(\mathcal{P}_{0}^{2}-\mathcal{P}_{2}^{2}\right)}{\left(\lambda^{1} R\right)^{4}} \sigma^{2}+\frac{\mathcal{P}_{2}^{2}}{\mathcal{P}_{0}^{2}-\mathcal{P}_{2}^{2}}
\end{aligned}
$$




$$
\begin{gathered}
X^{0}(\tau, r)_{T=0} \equiv t(\tau, r)_{T=0}=c_{1}\left[\tau+\frac{\sigma(r)}{\lambda^{1}}\right]-\frac{\mathcal{P}_{0}}{2} \int \frac{d r^{2}}{\left(r^{2}+1\right) \sqrt{\left(\mathcal{P}_{0}^{2}-\mathcal{P}_{2}^{2}\right) r^{2}-\mathcal{P}_{2}^{2}}}, \\
X^{2}(\tau, r)_{T=0} \equiv \phi(\tau, r)_{T=0}=-c_{1} \frac{\mathcal{P}_{0}}{\mathcal{P}_{2}}\left[\tau+\frac{\sigma(r)}{\lambda^{1}}\right]+\frac{\mathcal{P}_{2}}{2} \int \frac{d r^{2}}{r^{2} \sqrt{\left(\mathcal{P}_{0}^{2}-\mathcal{P}_{2}^{2}\right) r^{2}-\mathcal{P}_{2}^{2}}} ; \\
X^{0}(\tau, \sigma)_{T=0} \equiv t(\tau, \sigma)_{T=0}=c_{1}\left(\tau+\sigma / \lambda^{1}\right)-\frac{2 \lambda^{0} \mathcal{P}_{0}}{\left(\lambda^{1} R\right)^{2}} \int \frac{d \sigma}{r^{2}(\sigma)+1} \\
X^{2}(\tau, \sigma)_{T=0} \equiv \phi(\tau, \sigma)_{T=0}=-c_{1} \frac{\mathcal{P}_{0}}{\mathcal{P}_{2}}\left(\tau+\sigma / \lambda^{1}\right)+\frac{2 \lambda^{0} \mathcal{P}_{2}}{\left(\lambda^{1} R\right)^{2}} \int \frac{d \sigma}{r^{2}(\sigma)}
\end{gathered}
$$

\subsection{Obtaining new solutions}

Let us give an example, which is not discussed in the literature yet. To compare with what is known, let us recall that in 4], the string dynamics is considered in the background of $A d S_{5}$ black hole, with field theory dual finite temperature $\mathcal{N}=4 S Y M$. The background metric is taken to be

$$
\begin{aligned}
d s_{A d S_{5} B H}^{2}= & -\left(1+\frac{r^{2}}{R^{2}}-\frac{M}{r^{2}}\right) d t^{2}+\frac{d r^{2}}{\left(1+\frac{r^{2}}{R^{2}}-\frac{M}{r^{2}}\right)} \\
& +r^{2}\left(d \theta^{2}+\sin ^{2} \theta d \phi_{1}^{2}+\cos ^{2} \theta d \phi_{2}^{2}\right) .
\end{aligned}
$$

The classical string configuration, investigated in [4] in this gravitational field, is given by the ansatz

$$
t=\tau, \quad r=r(\sigma), \quad \theta=\frac{\pi}{2}, \quad \phi_{1}=\omega \tau, \quad \phi_{2}=0 .
$$

The background felt by the string for this embedding is

$$
d s^{2}=-\left(1+\frac{r^{2}}{R^{2}}-\frac{M}{r^{2}}\right) d t^{2}+\frac{d r^{2}}{\left(1+\frac{r^{2}}{R^{2}}-\frac{M}{r^{2}}\right)}+r^{2} d \phi_{1}^{2},
$$

and it depends on $x^{1}=r$ only. Therefore, $\mu=(0,2), a=1$ in our notation. (4.19) is particular case of (1.3), corresponding to

$$
\Lambda_{0}^{0}=1, \quad \Lambda_{0}^{2}=\omega, \quad \Lambda_{1}^{\mu}=0, \quad Z^{1}(\sigma)=r(\sigma) .
$$

Now, we are going to find new exact string solution, based on the ansatz (1.4), which corresponds to more general string embedding than (1.3). Moreover, none of the coordinates in (4.18) will be kept fixed. As far as the string background (4.18) depends on $x^{1}=r$ and $x^{2}=\theta, \mu=(0,3,4), a=(1,2)$ in our notation. In this case, the ansatz (1.4) reduces to (we take $\Lambda_{1}^{\mu}=0$ ):

$$
\begin{aligned}
& X^{0}(\tau, \sigma) \equiv t(\tau, \sigma)=\Lambda_{0}^{0} \tau+Z^{0}(\sigma), \\
& X^{1}(\tau, \sigma)=Z^{1}(\sigma)=r(\sigma) \\
& X^{2}(\tau, \sigma)=Z^{2}(\sigma)=\theta(\sigma) \\
& X^{3}(\tau, \sigma) \equiv \phi_{1}(\tau, \sigma)=\Lambda_{0}^{3} \tau+Z^{3}(\sigma), \\
& X^{4}(\tau, \sigma) \equiv \phi_{2}(\tau, \sigma)=\Lambda_{0}^{4} \tau+Z^{4}(\sigma) .
\end{aligned}
$$


The explicit expressions for the conserved momenta $P_{\mu}$ can be found from (3.5). By using them, one receives:

$$
\begin{aligned}
& E=-\int_{0}^{2 \pi} d \sigma P_{0}(\sigma)=-T \sqrt{-\gamma} \int_{0}^{2 \pi} d \sigma\left(1+\frac{r^{2}}{R^{2}}-\frac{M}{r^{2}}\right)\left(\gamma^{00} \Lambda_{0}^{0}+\gamma^{01} Z^{\prime 0}\right) \\
& S_{1}=\int_{0}^{2 \pi} d \sigma P_{3}(\sigma)=-T \sqrt{-\gamma} \int_{0}^{2 \pi} d \sigma r^{2} \sin ^{2} \theta\left(\gamma^{00} \Lambda_{0}^{3}+\gamma^{01} Z^{\prime 3}\right) \\
& S_{2}=\int_{0}^{2 \pi} d \sigma P_{4}(\sigma)=-T \sqrt{-\gamma} \int_{0}^{2 \pi} d \sigma r^{2} \cos ^{2} \theta\left(\gamma^{00} \Lambda_{0}^{4}+\gamma^{01} Z^{\prime 4}\right)
\end{aligned}
$$

The first integrals $\mathcal{P}_{\mu}$ for this part of the equations of motion (3.4), which correspond to $L=\mu$, are given by (3.6). Now, they simplify to:

$$
\mathcal{P}_{\mu}=-T \sqrt{-\gamma} g_{\mu \nu}\left(\gamma^{11} Z^{\prime \nu}+\gamma^{01} \Lambda_{0}^{\nu}\right)=\text { constants }
$$

The connection between the conserved momenta $P_{\mu}$ and the constants of the motion $\mathcal{P}_{\mu}$ following from (3.13), allows one to exclude $Z^{\prime \mu}$ from (4.21) and to rewrite $E, S_{1}$ and $S_{2}$ as follows

$$
\begin{aligned}
& E=-2 \pi \frac{\gamma^{01}}{\gamma^{11}} \mathcal{P}_{0}+\frac{T \Lambda_{0}^{0}}{\sqrt{-\gamma}} \int_{0}^{2 \pi} d \sigma\left(1+\frac{r^{2}}{R^{2}}-\frac{M}{r^{2}}\right), \\
& S_{1}=2 \pi \frac{\gamma^{01}}{\gamma^{11}} \mathcal{P}_{3}+\frac{T \Lambda_{0}^{3}}{\sqrt{-\gamma}} \int_{0}^{2 \pi} d \sigma r^{2} \sin ^{2} \theta, \\
& S_{2}=2 \pi \frac{\gamma^{01}}{\gamma^{11}} \mathcal{P}_{4}+\frac{T \Lambda_{0}^{4}}{\sqrt{-\gamma}} \int_{0}^{2 \pi} d \sigma r^{2} \cos ^{2} \theta .
\end{aligned}
$$

It is easy to check, that the above three quantities are constrained by the equality:

$$
\begin{aligned}
E= & -2 \pi \frac{\gamma^{01}}{\gamma^{11}}\left[\mathcal{P}_{0}+\frac{\Lambda_{0}^{0}}{R^{2}}\left(\frac{\mathcal{P}_{3}}{\Lambda_{0}^{3}}+\frac{\mathcal{P}_{4}}{\Lambda_{0}^{4}}\right)\right]+\frac{\Lambda_{0}^{0}}{R^{2}}\left(\frac{S_{1}}{\Lambda_{0}^{3}}+\frac{S_{2}}{\Lambda_{0}^{4}}\right) \\
& +\frac{2 \pi T \Lambda_{0}^{0}}{\sqrt{-\gamma}}\left(1-\frac{M}{2 \pi} \int_{0}^{2 \pi} \frac{d \sigma}{r^{2}(\sigma)}\right) .
\end{aligned}
$$

Let us now consider the compatibility condition (2.21). In the case at hand, it gives

$$
\Lambda_{0}^{4}=-\frac{1}{\mathcal{P}_{4}}\left(\Lambda_{0}^{0} \mathcal{P}_{0}+\Lambda_{0}^{3} \mathcal{P}_{3}\right)
$$

From now on, we will use this expression for $\Lambda_{0}^{4}$, thus ensuring that the constraint (3.3) is identically satisfied.

Our next goal is to solve the equations of motion (3.8) and the constraint (3.9). As explained in Sec.3, these equations have the same form as (2.24) and (2.22). Thus, for obtaining exact string solutions in the framework of the ansatz (1.4), we can use the formulas derived in Appendix A, after the replacements $(g, \Gamma, \mathcal{U}, \mathcal{A}) \rightarrow\left(h, \Gamma^{\mathbf{h}}, \mathcal{U}^{\mathbf{h}}, \mathcal{A}^{\mathbf{h}}\right)$. Since the background metric (4.18) is diagonal one and $b_{M N}=0$,

$$
h_{a b}=g_{a b}, \quad \Gamma_{a, b c}^{\mathbf{h}}=\Gamma_{a, b c}, \quad \mathcal{A}_{a}^{\mathbf{h}}=0 .
$$


So, we only need to replace $\mathcal{U} \rightarrow \mathcal{U}^{\mathbf{h}}$ and $\mathcal{A} \rightarrow \mathcal{A}^{\mathbf{h}}=0$ in (A.5) and (A.6). As far as the diagonal background in (4.18) depends on two coordinates, we can use the general expressions (A.11) and (A.13) for the first integrals of the equations (A.8), (A.9), which also solve the constraint (A.6), if the derived sufficient conditions (A.7), (A.10) and (A.12) are satisfied. Let us check this. The condition (A.7) does not appear, because in our case the effective gauge potential $\mathcal{A}^{\mathbf{h}}$ is identically zero. The first of the conditions (A.10) is satisfied, because it takes the form

$$
\frac{\partial}{\partial \theta}\left[\frac{g_{22}(r)}{g_{11}(r)}\right] \equiv 0
$$

Consequently, it remains to satisfy the second of the conditions (A.10) and the condition (A.12). In the case at hand, they require, the right hand sides of (A.11) and (A.13) to depend only on $Z^{2}=\theta$ and $Z^{1}=r$ respectively. To see if this is true, let us write down the first integrals (A.13) and (A.11) explicitly

$$
\begin{aligned}
& \left(g_{11} r^{\prime}\right)^{2}=-\frac{g_{11}(r)}{g_{22}(r)} D_{2}(r) \equiv F_{1}(r) \geq 0 \\
& \left(g_{22} \theta^{\prime}\right)^{2}=D_{2}(r)+g_{22}(r) \mathcal{U}^{\mathbf{h}}(r, \theta) \\
& =D_{2}(r)-\frac{1}{\gamma\left(\gamma^{11}\right)^{2}}\left\{\frac{\left(\Lambda_{0}^{0}\right)^{2}}{R^{2}} \Delta(r)-\left[\left(\Lambda_{0}^{3}\right)^{2} \sin ^{2} \theta+\frac{\left(\Lambda_{0}^{0} \mathcal{P}_{0}+\Lambda_{0}^{3} \mathcal{P}_{3}\right)^{2}}{\left(\mathcal{P}_{4}\right)^{2}} \cos ^{2} \theta\right] r^{4}\right. \\
& \left.+\frac{1}{T^{2}}\left[\frac{\mathcal{P}_{0}^{2} R^{2} r^{4}}{\Delta(r)}-\left(\frac{\mathcal{P}_{3}^{2}}{\sin ^{2} \theta}+\frac{\mathcal{P}_{4}^{2}}{\cos ^{2} \theta}\right)\right]\right\} \geq 0 \\
& \Delta(r)=r^{4}+R^{2}\left(r^{2}-M\right)
\end{aligned}
$$

It is evident that the r.h.s. of the equation for $r^{\prime}$ is a function only on $r$, while the r.h.s. of the equation for $\theta^{\prime}$ is not a function only on $\theta$. However, we have enough freedom to satisfy this last condition. To this end, we choose the arbitrary parameter $\Lambda_{0}^{3}$ and the arbitrary function $D_{2}(r)$ to be given by

$$
\begin{aligned}
& \Lambda_{0}^{3}=-\frac{\mathcal{P}_{0}}{\mathcal{P}_{3} \pm \mathcal{P}_{4}} \Lambda_{0}^{0} \Rightarrow \Lambda_{0}^{4}=-\frac{\mathcal{P}_{0}}{\mathcal{P}_{4}}\left(1-\frac{1}{\mathcal{P}_{3} \pm \mathcal{P}_{4}}\right) \Lambda_{0}^{0}, \\
& D_{2}(r)=c^{2}+\frac{1}{\gamma\left(\gamma^{11}\right)^{2}}\left[\frac{\left(\Lambda_{0}^{0}\right)^{2}}{R^{2}} \Delta(r)-\frac{\left(\Lambda_{0}^{0} \mathcal{P}_{0}\right)^{2}}{\left(\mathcal{P}_{3} \pm \mathcal{P}_{4}\right)^{2}} r^{4}+\frac{1}{T^{2}} \frac{\mathcal{P}_{0}^{2} R^{2} r^{4}}{\Delta(r)}\right] \leq 0,
\end{aligned}
$$

where $c$ is arbitrary constant. After this choice, the first integral of the equation of motion for the string coordinate $\theta(\sigma)$ takes the form

$$
\left(g_{22} \theta^{\prime}\right)^{2}=c^{2}+\frac{1}{\gamma\left(\gamma^{11}\right)^{2} T^{2}}\left(\frac{\mathcal{P}_{3}^{2}}{\sin ^{2} \theta}+\frac{\mathcal{P}_{4}^{2}}{\cos ^{2} \theta}\right) \equiv F_{2}(\theta) \geq 0 .
$$

In this way, all conditions are satisfied, and we can start solving the equations (4.23) and (4.25). The general solution of (4.23) is given by

$$
\sigma(r)=R^{2} \int \frac{r^{2} d r}{\Delta(r) \sqrt{F_{1}(r)}} .
$$


If we can obtain $r(\sigma)$ from here, the general solution of (4.25) will be

$$
\int \frac{d \theta}{\sqrt{F_{2}(\theta)}}=\int \frac{d \sigma}{r^{2}(\sigma)} .
$$

Anyway, we can always find the orbit $r=r(\theta)$ from (4.23) and (4.25), and it is defined by the equality

$$
R^{2} \int \frac{d r}{\Delta(r) \sqrt{F_{1}(r)}}=\int \frac{d \theta}{\sqrt{F_{2}(\theta)}} .
$$

To find the general solution for the remaining string coordinates $X^{\mu}$, we have to integrate the equations (3.7), which in our case simplify to

$$
Z^{\prime \mu}=-\frac{\gamma^{01}}{\gamma^{11}} \Lambda_{0}^{\mu}-\frac{\mathcal{P}_{\nu}}{T \sqrt{-\gamma} \gamma^{11}}\left(g^{-1}\right)^{\mu \nu}
$$

and then replace the received $Z^{\mu}$ into the initial ansatz (4.20). The same result may be obtained directly from (3.12) for solutions of the type $X^{\mu}(\tau, \sigma)$. Written explicitly, they are:

$$
\begin{aligned}
X^{0}(\tau, \sigma) \equiv t(\tau, \sigma)= & \Lambda_{0}^{0}\left(\tau-\frac{\gamma^{01}}{\gamma^{11}} \sigma\right)+\frac{R^{2} \mathcal{P}_{0}}{T \sqrt{-\gamma} \gamma^{11}} \int \frac{r^{2}(\sigma) d \sigma}{\Delta[r(\sigma)]} \\
X^{3}(\tau, \sigma) \equiv \phi_{1}(\tau, \sigma)= & -\frac{\Lambda_{0}^{0} \mathcal{P}_{0}}{\mathcal{P}_{3} \pm \mathcal{P}_{4}}\left(\tau-\frac{\gamma^{01}}{\gamma^{11}} \sigma\right)-\frac{\mathcal{P}_{3}}{T \sqrt{-\gamma} \gamma^{11}} \int \frac{d \sigma}{r^{2}(\sigma) \sin ^{2} \theta(\sigma)} \\
X^{4}(\tau, \sigma) \equiv \phi_{2}(\tau, \sigma)= & -\frac{\Lambda_{0}^{0} \mathcal{P}_{0}}{\mathcal{P}_{4}}\left(1-\frac{1}{\mathcal{P}_{3} \pm \mathcal{P}_{4}}\right)\left(\tau-\frac{\gamma^{01}}{\gamma^{11}} \sigma\right) \\
& -\frac{\mathcal{P}_{4}}{T \sqrt{-\gamma} \gamma^{11}} \int \frac{d \sigma}{r^{2}(\sigma) \cos ^{2} \theta(\sigma)}
\end{aligned}
$$

It is easy to take conformal gauge or the tensionless limit $T \rightarrow 0$ in the above solutions, and we will not consider these cases separately here.

As a final example, let us obtain a family of new string solutions in $A d S_{5} \times S^{5}$ background with two spins and up to nine independent conserved $R$-charges. We take the line elements in $A d S_{5}$ and on $S^{5}$ to be given by

$$
\begin{aligned}
& d s_{A d S_{5}}^{2}=R^{2}\left[-\cosh ^{2} \rho d t^{2}+d \rho^{2}+\sinh ^{2} \rho\left(d \theta^{2}+\sin ^{2} \theta d \phi^{2}+\cos ^{2} \theta d \varphi^{2}\right)\right], \\
& d s_{S^{5}}^{2}=R^{2}\left[d \gamma^{2}+\cos ^{2} \gamma d \varphi_{3}^{2}+\sin ^{2} \gamma\left(d \psi^{2}+\cos ^{2} \psi d \varphi_{1}^{2}+\sin ^{2} \psi d \varphi_{2}^{2}\right)\right] .
\end{aligned}
$$

Now, consider the following string embedding of type (1.3) (the angular coordinates $\theta$ and $\gamma$ are kept fixed to $\pi / 4$ )

$$
\begin{array}{ll}
X^{0}(\tau, \sigma) \equiv t(\tau, \sigma)=\Lambda_{0}^{0} \tau+\Lambda_{1}^{0} \sigma, & X^{4}(\tau, \sigma)=Z^{4}(\sigma)=\psi(\sigma) \\
X^{1}(\tau, \sigma)=Z^{1}(\sigma)=\rho(\sigma), & X^{5}(\tau, \sigma) \equiv \varphi_{1}(\tau, \sigma)=\Lambda_{0}^{5} \tau+\Lambda_{1}^{5} \sigma \\
X^{2}(\tau, \sigma) \equiv \phi(\tau, \sigma)=\Lambda_{0}^{2} \tau+\Lambda_{1}^{2} \sigma, & X^{6}(\tau, \sigma) \equiv \varphi_{2}(\tau, \sigma)=\Lambda_{0}^{6} \tau+\Lambda_{1}^{6} \sigma \\
X^{3}(\tau, \sigma) \equiv \varphi(\tau, \sigma)=\Lambda_{0}^{3} \tau+\Lambda_{1}^{3} \sigma, & X^{7}(\tau, \sigma) \equiv \varphi_{3}(\tau, \sigma)=\Lambda_{0}^{7} \tau+\Lambda_{1}^{7} \sigma \\
\mu=(0,2,3,5,6,7), \quad a=(1,4) . &
\end{array}
$$


The background metric seen by the string is

$$
\begin{aligned}
d s^{2} & =R^{2}\left[-\cosh ^{2} \rho d t^{2}+d \rho^{2}+\frac{1}{2} \sinh ^{2} \rho\left(d \phi^{2}+d \varphi^{2}\right)\right. \\
& \left.+\frac{1}{2}\left(d \psi^{2}+\cos ^{2} \psi d \varphi_{1}^{2}+\sin ^{2} \psi d \varphi_{2}^{2}+d \varphi_{3}^{2}\right)\right] .
\end{aligned}
$$

It does not depend on $x^{0}=t, x^{2}=\phi, x^{3}=\varphi, x^{5}=\varphi_{1}, x^{6}=\varphi_{2}, x^{7}=\varphi_{3}$, so the Lagrangian density (2.11) does not depend on $X^{\mu}(\tau, \sigma), \mu=(0,2,3,5,6,7)$. Consequently, the corresponding generalized momenta (2.16) are conserved

$$
\begin{gathered}
p_{\mu}=\int_{0}^{2 \pi} d \sigma P_{\mu}(\sigma)=\text { constants, } \\
p_{0} \equiv-E=R^{2} T \sqrt{-\gamma} \gamma^{0 n} \Lambda_{n}^{0} \int_{0}^{2 \pi} d \sigma \cosh ^{2} \rho(\sigma), \\
p_{2} \equiv S_{1}=-\frac{R^{2} T}{2} \sqrt{-\gamma} \gamma^{0 n} \Lambda_{n}^{2} \int_{0}^{2 \pi} d \sigma \sinh ^{2} \rho(\sigma), \\
p_{3} \equiv S_{2}=-\frac{R^{2} T}{2} \sqrt{-\gamma} \gamma^{0 n} \Lambda_{n}^{3} \int_{0}^{2 \pi} d \sigma \sinh ^{2} \rho(\sigma), \\
p_{5} \equiv J_{1}=-\frac{R^{2} T}{2} \sqrt{-\gamma} \gamma^{0 n} \Lambda_{n}^{5} \int_{0}^{2 \pi} d \sigma \cos ^{2} \psi(\sigma), \\
p_{6} \equiv J_{2}=-\frac{R^{2} T}{2} \sqrt{-\gamma} \gamma^{0 n} \Lambda_{n}^{6} \int_{0}^{2 \pi} d \sigma \sin ^{2} \psi(\sigma), \\
p_{7} \equiv J_{3}=-\pi R^{2} T \sqrt{-\gamma} \gamma^{0 n} \Lambda_{n}^{7} .
\end{gathered}
$$

In addition, there exist the constants of the motion (2.20), which must satisfy the condition (2.21). In our case, we may choose for instance

$$
\Lambda_{0}^{3}=-\frac{1}{\mathcal{P}_{3}}\left(\Lambda_{0}^{0} \mathcal{P}_{0}+\Lambda_{0}^{2} \mathcal{P}_{2}+\Lambda_{0}^{5} \mathcal{P}_{5}+\Lambda_{0}^{6} \mathcal{P}_{6}+\Lambda_{0}^{7} \mathcal{P}_{7}\right),
$$

to ensure that the constraint (2.14) is identically fulfilled.

The first integrals (A.13), (A.11), of the equations of motion for $\rho(\sigma)$ and $\psi(\sigma)$, which also solve the constraint (A.6), read

$$
\begin{aligned}
& \left(g_{11} \rho^{\prime}\right)^{2}=-2 D_{4}(\rho) \equiv F_{1}(\rho) \geq 0 \\
& \left(g_{44} \psi^{\prime}\right)^{2}=D_{4}(\rho)+g_{44} \mathcal{U}(\rho, \psi) \\
& =d+\frac{R^{4}}{4 \gamma^{11}}\left(C^{55} \cos ^{2} \psi+C^{66} \sin ^{2} \psi+C^{77}\right) \equiv F_{4}(\psi) \geq 0 \\
& d=\text { const }, \quad C^{\mu \nu}=\gamma^{m n} \Lambda_{m}^{\mu} \Lambda_{n}^{\nu} .
\end{aligned}
$$

Here, the arbitrary function $D_{4}(\rho)$ has been chosen to be given by

$$
D_{4}(\rho)=d-\frac{R^{4}}{2 \gamma^{11}}\left[\frac{2 \Lambda_{1}^{\mu} \mathcal{P}_{\mu}}{R^{2} T \sqrt{-\gamma}}-C^{00} \cosh ^{2} \rho+\frac{1}{2}\left(C^{22}+C^{33}\right) \sinh ^{2} \rho\right] \leq 0,
$$


in order the integrability condition (A.12) to be satisfied ${ }^{7}$. The general solutions of the differential equations (4.30) and (4.31), for the string coordinates $\rho(\sigma)$ and $\psi(\sigma)$, are

$$
\sigma(\rho)=R^{2} \int \frac{d \rho}{\sqrt{F_{1}(\rho)}}, \quad \sigma(\psi)=\frac{R^{2}}{2} \int \frac{d \psi}{\sqrt{F_{4}(\psi)}} .
$$

From (4.30) and (4.31), one can also find the orbit $\rho=\rho(\psi)$

$$
2 \int \frac{d \rho}{\sqrt{F_{1}(\rho)}}=\int \frac{d \psi}{\sqrt{F_{4}(\psi)}} .
$$

Now, let us show that for the string solution obtained above, there exist other nontrivial conserved angular momenta on $S^{5}$ (R-charges), besides $p_{5}=J_{1}, p_{6}=J_{2}$ and $p_{7}=J_{3}$. To this aim, following [24, we introduce new embedding coordinates on $S^{5}$, working in conformal gauge

$$
\begin{aligned}
& W_{1}+i W_{2}=R \sin \gamma \cos \psi e^{i \varphi_{1}}, \quad W_{3}+i W_{4}=R \sin \gamma \sin \psi e^{i \varphi_{2}}, \\
& W_{5}+i W_{6}=R \cos \gamma e^{i \varphi_{3}}, \quad \sum_{A=1}^{6} W_{A}^{2}=R^{2} .
\end{aligned}
$$

In terms of these coordinates, the generators of the $S^{5}$ isometry group $O(6)$ are $\left(\partial_{0}=\right.$ $\partial / \partial \tau)$

$$
J_{A B}=T \int_{0}^{2 \pi} d \sigma\left(W_{A} \partial_{0} W_{B}-W_{B} \partial_{0} W_{A}\right)
$$

and they must be conserved in time: $\partial_{0} J_{A B}=0$.

For our ansatz (4.28), the coordinates $W_{A}$ acquire the form

$$
\begin{aligned}
& W_{1}(\tau, \sigma)=\frac{R}{\sqrt{2}} \cos \psi(\sigma) \cos \left(\Lambda_{0}^{5} \tau+\Lambda_{1}^{5} \sigma\right), W_{2}(\tau, \sigma)=\frac{R}{\sqrt{2}} \cos \psi(\sigma) \sin \left(\Lambda_{0}^{5} \tau+\Lambda_{1}^{5} \sigma\right), \\
& W_{3}(\tau, \sigma)=\frac{R}{\sqrt{2}} \sin \psi(\sigma) \cos \left(\Lambda_{0}^{6} \tau+\Lambda_{1}^{6} \sigma\right), W_{4}(\tau, \sigma)=\frac{R}{\sqrt{2}} \sin \psi(\sigma) \sin \left(\Lambda_{0}^{6} \tau+\Lambda_{1}^{6} \sigma\right), \\
& W_{5}(\tau, \sigma)=\frac{R}{\sqrt{2}} \cos \left(\Lambda_{0}^{7} \tau+\Lambda_{1}^{7} \sigma\right), W_{6}(\tau, \sigma)=\frac{R}{\sqrt{2}} \sin \left(\Lambda_{0}^{7} \tau+\Lambda_{1}^{7} \sigma\right) .
\end{aligned}
$$

By using these expressions for $W_{A}$, it is easy to check that $J_{12}, J_{34}$ and $J_{56}$ coincide with the conserved momenta (4.29), taken in conformal gauge

$$
\begin{aligned}
& J_{12}=p_{5}^{c g}=\frac{R^{2} T}{2} \Lambda_{0}^{5} \int_{0}^{2 \pi} d \sigma \cos ^{2} \psi(\sigma), \\
& J_{34}=p_{6}^{c g}=\frac{R^{2} T}{2} \Lambda_{0}^{6} \int_{0}^{2 \pi} d \sigma \sin ^{2} \psi(\sigma), \\
& J_{56}=p_{7}^{c g}=\pi R^{2} T \Lambda_{0}^{7} .
\end{aligned}
$$

\footnotetext{
${ }^{7}$ The condition (A.7) does not appear, because $\mathcal{A}_{a}=0$. The remaining conditions A.10) are satisfied identically.
} 
The conservation conditions $\partial_{0} J_{A B}=0$, applied to the remaining angular momenta $J_{A B}$, constrain the parameters $\Lambda_{0}^{5}, \Lambda_{0}^{6}$ and $\Lambda_{0}^{7}$ as follows

$$
\Lambda_{0}^{5}=\Lambda_{0}^{7}=\omega, \quad \Lambda_{0}^{6}= \pm \omega .
$$

Taking this into account, one obtains the following expressions for the other generators $J_{A B}$

$$
\begin{aligned}
J_{13}^{ \pm} & = \pm J_{24}^{ \pm}=\frac{R^{2} T}{2} \omega \int_{0}^{2 \pi} d \sigma\left[\sin \left(\omega \tau+\Lambda_{1}^{5} \sigma\right) \cos \left( \pm \omega \tau+\Lambda_{1}^{6} \sigma\right)\right. \\
& \left.\mp \sin \left( \pm \omega \tau+\Lambda_{1}^{6} \sigma\right) \cos \left(\omega \tau+\Lambda_{1}^{5} \sigma\right)\right] \sin \psi(\sigma) \cos \psi(\sigma) \\
J_{14}^{ \pm} & =\mp J_{23}^{ \pm}=\frac{R^{2} T}{2} \omega \int_{0}^{2 \pi} d \sigma\left[\sin \left(\omega \tau+\Lambda_{1}^{5} \sigma\right) \sin \left( \pm \omega \tau+\Lambda_{1}^{6} \sigma\right)\right. \\
& \left. \pm \cos \left(\omega \tau+\Lambda_{1}^{5} \sigma\right) \cos \left( \pm \omega \tau+\Lambda_{1}^{6} \sigma\right)\right] \sin \psi(\sigma) \cos \psi(\sigma) \\
J_{15} & =J_{26}=\frac{R^{2} T}{2} \omega \int_{0}^{2 \pi} d \sigma\left[\sin \left(\omega \tau+\Lambda_{1}^{5} \sigma\right) \cos \left(\omega \tau+\Lambda_{1}^{7} \sigma\right)\right. \\
& \left.-\sin \left(\omega \tau+\Lambda_{1}^{7} \sigma\right) \cos \left(\omega \tau+\Lambda_{1}^{5} \sigma\right)\right] \cos \psi(\sigma) \\
J_{16} & =-J_{25}=\frac{R^{2} T}{2} \omega \int_{0}^{2 \pi} d \sigma\left[\sin \left(\omega \tau+\Lambda_{1}^{5} \sigma\right) \sin \left(\omega \tau+\Lambda_{1}^{7} \sigma\right)\right. \\
& \left.+\cos \left(\omega \tau+\Lambda_{1}^{5} \sigma\right) \cos \left(\omega \tau+\Lambda_{1}^{7} \sigma\right)\right] \cos \psi(\sigma) \\
J_{35}^{ \pm} & = \pm J_{46}^{ \pm}= \pm \frac{R^{2} T}{2} \omega \int_{0}^{2 \pi} d \sigma\left[\sin \left( \pm \omega \tau+\Lambda_{1}^{6} \sigma\right) \cos \left(\omega \tau+\Lambda_{1}^{7} \sigma\right)\right. \\
& \left.\mp \sin \left(\omega \tau+\Lambda_{1}^{7} \sigma\right) \cos \left( \pm \omega \tau+\Lambda_{1}^{6} \sigma\right)\right] \sin \psi(\sigma) \\
J_{36}^{ \pm} & =\mp J_{45}^{ \pm}= \pm \frac{R^{2} T}{2} \omega \int_{0}^{2 \pi} d \sigma\left[\sin \left( \pm \omega \tau+\Lambda_{1}^{6} \sigma\right) \sin \left(\omega \tau+\Lambda_{1}^{7} \sigma\right)\right. \\
& \left. \pm \cos \left( \pm \omega \tau+\Lambda_{1}^{6} \sigma\right) \cos \left(\omega \tau+\Lambda_{1}^{7} \sigma\right)\right] \sin \psi(\sigma) .
\end{aligned}
$$

Hence, our string solution characterizes with nine independent conserved $R$-charges, if $\Lambda_{1}^{5}$, $\Lambda_{1}^{6}, \Lambda_{1}^{7}$, are all different.

\section{Concluding remarks}

The string embeddings (1.1) - (1.4) allow us to reduce the problem of solving the string equations of motion and constraints to a particle-like one. To achieve this, one have to get rid of the dependence on the spatial worldsheet coordinate $\sigma$, or on the temporal worldsheet coordinate $\tau$. To this aim, since the string action contains the first derivatives $\partial_{m} X^{M}$, the string coordinates $X^{M}(\tau, \sigma)$ must depend on $\sigma$ or $\tau$ at most linearly. Besides, the background fields entering the action depend implicitly on $\tau$ and $\sigma$ through their dependence on $X^{M}$. If we accept that the external fields depend only on part of the coordinates, say $X^{a}$, the resulting reduced Lagrangian density will depend only on $\xi^{0}=\tau$ for the ansatzes (1.1) and (1.2), or on $\xi^{1}=\sigma$ for the ansatzes (1.3) and (1.4). 
Of course, one can use different approach to simplify the problem of solving the nonlinear string equations of motion in variable external fields [16, 27]. The two ansatzes used in [16, which do not belong to the types (1.1) - (1.4), are the following

$$
t=t(\tau), \quad r=f(\tau) g(\sigma), \quad l=F(\tau) G(\sigma), \quad \phi=\omega \tau
$$

and

$$
t=t(\tau), \quad r=r(\tau), \quad \theta=\theta(\sigma), \quad \varphi=\nu \tau
$$

The backgrounds do not depend on $(t, \phi)$ and on $(t, \varphi)$ respectively. The three ansatzes, used in [27], are

$$
\begin{aligned}
& t=c_{0} \tau, \quad r=r(\sigma), \quad \phi=\omega \tau, \quad \theta=\theta(\tau), \quad \psi=\sigma, \\
& t=t(\tau), \quad r=r(\tau), \quad \phi=\sigma, \quad \theta=\theta(\sigma), \quad \psi=\nu \tau, \\
& t=c_{0} \tau, \quad \beta=\sigma, \quad \phi=\omega \tau, \quad \tilde{\phi}=\omega \tau, \quad \theta=\theta(\tau), \quad \psi=\sigma .
\end{aligned}
$$

The corresponding background metrics seen by the string do not depend on $(t, \phi, \psi)$ in the first two cases and on $(t, \phi, \tilde{\phi}, \psi)$ in the third one. Therefore, all the ansatzes used in the literature, are particular cases of the following string embedding

$$
X^{\mu}(\tau, \sigma)=\Lambda_{0}^{\mu} \tau+\Lambda_{1}^{\mu} \sigma+Y^{\mu}(\tau) Z^{\mu}(\sigma), \quad X^{a}(\tau, \sigma)=Y^{a}(\tau) Z^{a}(\sigma) .
$$

Let us also mention that the ansatz of the type $X(\tau, \sigma)=Y(\tau) Z(\sigma)$, used in [24], is equivalent to (1.3) with $\Lambda_{1}^{\mu}=0$, after change of the embedding coordinates ${ }^{8}$.

Our task in this paper was to find as more general string solutions as we can. That is why, we do not consider the different periodicity and normalization conditions, which arise for particular string configurations. However, once the general solution is found, they can be always implemented.

\section{Acknowledgments}

This work is supported by Shoumen University grants under contracts No.001/2003 and No.003/2004.

\section{Appendix A}

Here, we will explain how to find solutions of the string equations of motion (2.24) and of the effective constraint (2.22).

Let us start with the simplest case, when the background fields depend on only one coordinate $X^{a}(\tau, \sigma)=Z^{a}(\sigma)$. In this case the Eqs. (2.24), (2.22) simplify to

$$
\begin{aligned}
& \frac{d}{d \sigma}\left(g_{a a} Z^{\prime a}\right)-\frac{1}{2} \frac{d g_{a a}}{d Z^{a}}\left(Z^{\prime a}\right)^{2}=\frac{1}{2} \frac{d \mathcal{U}}{d Z^{a}}, \\
& g_{a a}\left(Z^{\prime a}\right)^{2}=\mathcal{U},
\end{aligned}
$$

\footnotetext{
${ }^{8}$ See the last example in the previous section.
} 
where we have used that

$$
g_{a b} Z^{\prime \prime b}+\Gamma_{a, b c} Z^{\prime b} Z^{\prime c}=\frac{d}{d \sigma}\left(g_{a b} Z^{\prime b}\right)-\frac{1}{2} \partial_{a} g_{b c} Z^{\prime b} Z^{\prime c} .
$$

After multiplying with $2 g_{a a} Z^{\prime a}$ and after using the constraint (A.2), the Eq. (A.1) reduces to

$$
\frac{d}{d \sigma}\left[\left(g_{a a} Z^{\prime a}\right)^{2}-g_{a a} \mathcal{U}\right]=0
$$

The solution of (A.3), compatible with (A.2), is just the constraint (A.2). In other words, (A.2) is first integral of the equation for the coordinate $Z^{a}$. By integrating (A.2), one obtains the following exact string solution

$$
\sigma\left(X^{a}\right)=\sigma_{0}+\int_{X_{0}^{a}}^{X^{a}}\left(\frac{\mathcal{U}}{g_{a a}}\right)^{-1 / 2} d x
$$

where $\sigma_{0}$ and $X_{0}^{a}$ are arbitrary constants.

Let us turn to the more complicated case, when the background fields depend on more than one coordinate $X^{a}(\tau, \sigma)=Z^{a}(\sigma)$. We would like to apply the same procedure for solving the system of differential equations (2.24), (2.22), as in the simplest case just considered. To be able to do this, we need to suppose that the metric $g_{a b}$ is a diagonal one. Then one can rewrite the effective equations of motion (2.24) and the effective constraint (2.22) in the form

$$
\begin{aligned}
& \frac{d}{d \sigma}\left(g_{a a} Z^{\prime a}\right)^{2}-Z^{\prime a} \partial_{a}\left(g_{a a} \mathcal{U}\right) \\
& +Z^{\prime a} \sum_{b \neq a}\left[\partial_{a}\left(\frac{g_{a a}}{g_{b b}}\right)\left(g_{b b} Z^{\prime b}\right)^{2}-4 \partial_{[a} \mathcal{A}_{b]} g_{a a} Z^{\prime b}\right]=0 \\
& g_{a a}\left(Z^{\prime a}\right)^{2}+\sum_{b \neq a} g_{b b}\left(Z^{\prime b}\right)^{2}=\mathcal{U} .
\end{aligned}
$$

To find solutions of the above equations without choosing particular background, we can fix all coordinates $X^{a}$ except one. Then the exact string solution is given again by the same expression (A.4) for $\sigma\left(X^{a}\right)$.

To find solutions depending on more than one coordinate, we must impose further conditions on the background fields. Let us show, how a number of sufficient conditions, which allow us to reduce the order of the equations of motion by one, can be obtained.

First of all, we split the index $a$ in such a way that $Z^{r}$ is one of the coordinates $Z^{a}$, and $Z^{\alpha}$ are the others. Then we assume that the effective 1 -form gauge field $\mathcal{A}_{a}$ can be represented in the form

$$
\mathcal{A}_{a} \equiv\left(\mathcal{A}_{r}, \mathcal{A}_{\alpha}\right)=\left(\mathcal{A}_{r}, \partial_{\alpha} f\right)
$$

i.e., it is oriented along the coordinate $Z^{r}$, and the remaining components $\mathcal{A}_{\alpha}$ are pure gauges. Now, the Eqs. A.5 read

$$
\frac{d}{d \sigma}\left(g_{\alpha \alpha} Z^{\prime \alpha}\right)^{2}-Z^{\prime \alpha} \partial_{\alpha}\left(g_{\alpha \alpha} \mathcal{U}\right)
$$




$$
\begin{aligned}
& +Z^{\prime \alpha}\left[\partial_{\alpha}\left(\frac{g_{\alpha \alpha}}{g_{r r}}\right)\left(g_{r r} Z^{\prime r}\right)^{2}-2 g_{\alpha \alpha} \partial_{\alpha}\left(\mathcal{A}_{r}-\partial_{r} f\right) Z^{\prime r}\right] \\
& +Z^{\prime \alpha} \sum_{\beta \neq \alpha} \partial_{\alpha}\left(\frac{g_{\alpha \alpha}}{g_{\beta \beta}}\right)\left(g_{\beta \beta} Z^{\prime \beta}\right)^{2}=0, \\
& \frac{d}{d \sigma}\left(g_{r r} Z^{\prime r}\right)^{2}-Z^{\prime r} \partial_{r}\left(g_{r r} \mathcal{U}\right) \\
& +Z^{\prime r} \sum_{\alpha}\left[\partial_{r}\left(\frac{g_{r r}}{g_{\alpha \alpha}}\right)\left(g_{\alpha \alpha} Z^{\prime \alpha}\right)^{2}+2 g_{r r} \partial_{\alpha}\left(\mathcal{A}_{r}-\partial_{r} f\right) Z^{\prime \alpha}\right]=0 .
\end{aligned}
$$

After imposing the conditions

$$
\partial_{\alpha}\left(\frac{g_{\alpha \alpha}}{g_{a a}}\right)=0, \quad \partial_{\alpha}\left(g_{r r} Z^{\prime r}\right)^{2}=0
$$

the Eqs. (A.8) reduce to

$$
\frac{d}{d \sigma}\left(g_{\alpha \alpha} Z^{\prime \alpha}\right)^{2}-Z^{\prime \alpha} \partial_{\alpha}\left\{g_{\alpha \alpha}\left[\mathcal{U}+2\left(\mathcal{A}_{r}-\partial_{r} f\right) Z^{\prime r}\right]\right\}=0,
$$

which are solved by

$$
\left(g_{\alpha \alpha} Z^{\prime \alpha}\right)^{2}=D_{\alpha}\left(Z^{a \neq \alpha}\right)+g_{\alpha \alpha}\left[\mathcal{U}+2\left(\mathcal{A}_{r}-\partial_{r} f\right) Z^{\prime r}\right]=F_{\alpha}\left(Z^{\beta}\right) \geq 0,
$$

where $D_{\alpha}, F_{\alpha}$ are arbitrary functions of their arguments. ${ }^{9}$

To integrate the Eq. (A.9), we impose the condition

$$
\partial_{r}\left(g_{\alpha \alpha} Z^{\prime \alpha}\right)^{2}=0 .
$$

After using the second of the conditions (A.10), the condition (A.12), and the already obtained solution (A.11), the Eq. (A.9) can be recast in the form

$$
\begin{aligned}
& \frac{d}{d \sigma}\left[\left(g_{r r} Z^{\prime r}\right)^{2}+2 g_{r r}\left(\mathcal{A}_{r}-\partial_{r} f\right) Z^{\prime r}\right] \\
& =Z^{\prime r} \partial_{r}\left\{g_{r r}\left[\left(1-n_{\alpha}\right)\left(\mathcal{U}+2\left(\mathcal{A}_{r}-\partial_{r} f\right) Z^{\prime r}\right)-\sum_{\alpha} \frac{D_{\alpha}\left(Z^{a \neq \alpha}\right)}{g_{\alpha \alpha}}\right]\right\},
\end{aligned}
$$

where $n_{\alpha}$ is the number of the coordinates $Z^{\alpha}$. The solution of this equation, compatible with (A.11) and with the effective constraint (A.6), is

$$
\left(g_{r r} Z^{\prime r}\right)^{2}=g_{r r}\left[\left(1-n_{\alpha}\right) \mathcal{U}-2 n_{\alpha}\left(\mathcal{A}_{r}-\partial_{r} f\right) Z^{\prime r}-\sum_{\alpha} \frac{D_{\alpha}\left(Z^{a \neq \alpha}\right)}{g_{\alpha \alpha}}\right]=F_{r}\left(Z^{r}\right) \geq 0(
$$

where $F_{r}$ is again an arbitrary function.

Thus, we succeeded to separate the variables $Z^{\prime a}$ and to obtain the first integrals (A.11), (A.13) for the equations of motion (A.5), when the conditions (A.7), (A.10),

\footnotetext{
${ }^{9} F_{\alpha}=F_{\alpha}\left(Z^{\beta}\right)$ follows from (A.12).
} 
(A.12) on the background fields are fulfilled. Further progress is possible, when working with particular background configurations, allowing for separation of the variables in (A.11) and (A.13).

Let us finally point out that (A.7), A.10) and (A.12) are just an example of possible integrability conditions, which are fulfilled in many cases, but in many others - they are not.

\section{References}

[1] S. S. Gubser, I. R. Klebanov, A. M. Polyakov, A semi-classical limit of the gauge/string correspondence, Nucl. Phys. B 636 (2002) 99 hep-th/0204051.

[2] S. Frolov, A.A. Tseytlin, Semiclassical quantization of rotating superstring in $A d S_{5} \times$ $S^{5}$, JHEP 0206 (2002) 007 hep-th/0204226.

[3] J. G. Russo, Anomalous dimensions in gauge theories from rotating strings in $A d S_{5} \times$ $S^{5}$, JHEP 0206 (2002) 038 hep-th/0205244.

[4] A. Armoni, L. J. F. Barbon, A. C. Petkou, Orbiting strings in AdS black holes and $\mathcal{N}=4$ SYM at finite temperature, JHEP 0206 (2002) 058 hep-th/0205280.

[5] G. Mandal, N. V. Suryanarayana, S. R. Wadia, Aspects of semiclassical strings in $A d S_{5}$, Phys. Lett. B 543 (2002) 81 hep-th/0206103.

[6] J. A. Minahan, Circular semiclassical string solutions on $A d S_{5} \times S^{5}$, Nucl. Phys. B 648 (2003) 203 hep-th/0209047.

[7] A. A. Tseytlin, Semiclassical quantization of superstrings: $A d S_{5} \times S^{5}$ and beyond, Int. J. Mod. Phys. A 18 (2003) 981 hep-th/0209116.

[8] A. Armoni, L. J. F. Barbon, A. C. Petkou, Rotating strings in confining AdS/CFT backgrounds, JHEP 0210 (2002) 069 hep-th/0209224.

[9] M. Alishahiha, A. E. Mosaffa, Circular semiclassical string solutions on confining AdS/CFT backgrounds, JHEP 0210 (2002) 060 hep-th/0210122.

[10] S. A. Hartnoll, C. Nunez, Rotating membranes on $G_{2}$ manifolds, logarithmic anomalous dimensions and $\mathcal{N}=1$ duality, JHEP 0302 (2003) 049 hep-th/0210218.

[11] A. Buchel, Gauge/string correspondence in curved space, Phys. Rev. D 67 (2003) 066004 hep-th/0211141.

[12] R. C. Rashkov, K. S. Viswanathan, Rotating strings with B-field, hep-th/0211197.

[13] A. Loewi, Y. Oz, Large spin strings in $A d S_{3}$, Phys. Lett. B 557 (2003) 253 hep-th/0212147.

[14] J. M. Pons, P. Talavera, Semiclassical string solutions for $\mathcal{N}=1 S Y M$, Nucl. Phys. B 665 (2003) 129 hep-th/0301178. 
[15] M. Alishahiha, A. E. Mosaffa, Semiclassical string solutions on deformed NS5-brane backgrounds and new plane wave, hep-th/0302005.

[16] S. Ryang, Rotating and orbiting strings in the near-horizon brane backgrounds, JHEP 0304 (2003) 045 hep-th/0303237.

[17] Martin Kruczenski, David Mateos, Robert C. Myers, David J. Winters, Meson spectroscopy in AdS/CFT with flavour, JHEP 0307 (2003) 049 hep-th/0304032.

[18] H. Dimov, V. Filev, R. C. Rashkov, K. S. Viswanathan, Semiclassical quantization of rotating strings in Pilch-Warner geometry, Phys. Rev. D 68 (2003) 066010 hep-th/0304035.

[19] S. Frolov, A. A. Tseytlin, Multi-spin string solutions in $A d S_{5} \times S^{5}$, Nucl. Phys. B 668 (2003) 77 hep-th/0304255.

[20] P. Matlock, K. S. Viswanathan, Y. Yang, R.Parthasarathy, NS5-brane and little string duality in the pp-wave limit, Phys. Rev. D 68 (2003) 086001 hep-th/0305028.

[21] A. L. Larsen, M. A. Lomholt, Open string fluctuations in AdS with and without torsion, Phys. Rev. D 68 (2003) 066002 hep-th/0305034.

[22] L. A. P. Zayas, D. Vaman, Hadronic density of states from string theory, Phys. Rev. Lett. 91 (2003) 111602 hep-th/0306107.

[23] S. Frolov, A. A. Tseytlin, Rotating string solutions: AdS/CFT duality in nonsupersymmetric sectors, Phys. Lett. B 570 (2003) 96 hep-th/0306143.

[24] G. Arutyunov, S. Frolov, J. Russo, A.A. Tseytlin, Spinning strings in $A d S_{5} \times S^{5}$ and integrable systems, Nucl. Phys. B 671 (2003) 3 hep-th/0307191.

[25] N. Besert, S. Frolov, M. Staudacher, A.A. Tseytlin, Precision spectroscopy of AdS/CFT, JHEP 0310 (2003) 037 hep-th/0308117.

[26] M. Schvellinger, Spinning and rotating strings for $\mathcal{N}=1 S Y M$ theory and brane constractions, JHEP 0402 (2004) 066 hep-th/0309161.

[27] A. Khan, A.L. Larsen, Spinning pulsating string solitons in $A d S_{5} \times S^{5}$, Phys. Rev. D 69 (2004) 026001 hep-th/0310019.

[28] A.L. Larsen, N. Sanchez, Strings propagating in the $2+1$ dimensional black hole AdS spacetime, Phys. Rev. D 50 (1994) 7493 hep-th/9405026;

A.L. Larsen, N. Sanchez, Mass spectrum of strings in AdS spacetime, Phys. Rev. D 52 (1995) 1051 hep-th/9410132;

H. de Vega, A.L. Larsen, N. Sanchez, Semi-classical quantization of circular strings in $d S$ and $A d S$ spacetimes, Phys. Rev. D 51 (1995) 6917 hep-th/9410219;

A.L. Larsen, N. Sanchez, New classes of exact multi-string solutions in curved spacetimes, Phys. Rev. D 51 (1995) 6929 hep-th/9501101;

A.L. Larsen, N. Sanchez, Sinh - Gordon, cosh - Gordon and Liouville equations for 
strings and multistrings in constant curvature spacetimes, Phys. Rev. D 54 (1996) 2801 hep-th/9606049;

H. de Vega, I. Egusquiza, Planetoid string solutions in $3+1$ axisymmetric spacetimes, Phys. Rev. D 54 (1996) 7513 hep-th/9607056;

H. de Vega, A.L. Larsen, N. Sanchez, Quantum string dynamics in the conformal invariant $S L(2, R) W Z W N$ background: AdS space with torsion, Phys. Rev. D 58 (1998) 026001 hep-th/9803038;

A.L. Larsen, N. Sanchez, From WZWN model to the Liouville equation: exact string dynamics in conformally invariant AdS background, Phys. Rev. D 58 (1998) 126002 hep-th/9805173;

A.L. Larsen, N. Sanchez, Quantum coherent string states in $A d S_{3}$ and $S L(2, R) W Z W N$ model, Phys. Rev. D 62 (2000) 046003 hep-th/0001180;

A.L. Larsen, N. Sanchez, New coherent string states and minimal uncertainty in WZWN models, Nucl. Phys. B 618 (2001) 301 hep-th/0103044.

[29] P. Bozhilov, Exact string solutions in nontrivial backgrounds, Phys. Rev. D 65 (2002) 026004 hep-th/0103154.

[30] N. Sanchez, Advances in string theory in curved backgrounds: a synthesis report, Int. J. Mod. Phys. A 18 (2003) 2011 hep-th/0302228.

[31] D. Aleksandrova, P. Bozhilov, On the classical string solutions and string/field theory duality, JHEP 0308 (2003) 018 hep-th/0307113.

[32] J. Isberg, U.Lindstrom, B. Sundborg, G. Theodoridis, Classical and quantized tensionless strings, Nucl. Phys. B 411 (1994) 122, hep-th/9307108.

[33] S. Hassani, U. Lindstrom, R. von Unge, Classicaly equivalent actions for tensionless P-branes, Class. Quant. Grav. 11 (1994) L79. 